\title{
The $\mathrm{pK}^{0} \Sigma^{+}$final state in proton-proton collisions
}

\author{
The COSY-TOF Collaboration
}

M. Abdel-Bary ${ }^{3}$, S. Abdel-Samad ${ }^{3}$, K-Th. Brinkmann ${ }^{1,8}$, H. Clement ${ }^{4}$, J. Dietrich ${ }^{1}$, E. Doroshkevich ${ }^{4}$, S. Dshemuchadse ${ }^{1}$, K. Ehrhardt ${ }^{4}$, A. Erhardt ${ }^{4}$, W. Eyrich ${ }^{2}$, A. Filippi ${ }^{7}$, H. Freiesleben ${ }^{1}$, M. Fritsch $^{2}$, W. Gast $^{3}$, J. Georgi ${ }^{2}$, A. Gillitzer ${ }^{3}$, J. Gottwald ${ }^{1}$, D. Hesselbarth ${ }^{3}$, H. Jäger ${ }^{3}$, B. Jakob ${ }^{1}$, R. Jäkel ${ }^{1}$, L. Karsch ${ }^{1}$, K. Kilian ${ }^{3}$, H. Koch ${ }^{9}$, M. Krapp ${ }^{2}$, J. Kreß ${ }^{4}$, E. Kuhlmann ${ }^{1}$, A. Lehmann ${ }^{2}$, S. Marcello ${ }^{7}$, S. Marwinski ${ }^{3}$, S. Mauro $^{9}$, W. Meyer $^{9}$, P. Michel ${ }^{5}$, K. Möller ${ }^{5}$, H.P. Morsch ${ }^{6}$, H. Mörtel ${ }^{2}$, L. Naumann ${ }^{5}$, N. Paul ${ }^{3}$, L. Pinna ${ }^{2}$, C. Pizzolotto ${ }^{2}$, Ch. Plettner ${ }^{1}$, S. Reimann ${ }^{1}$, M. Richter ${ }^{1}$, J. Ritman ${ }^{3}$, E. Roderburg ${ }^{3}$, A. Schamlott ${ }^{5}$, P. Schönmeier ${ }^{1}$, W. Schroeder ${ }^{2,3}$, M. Schulte-Wissermann ${ }^{1, a}$, T. Sefzick ${ }^{3}$, F. Stinzing ${ }^{2}$, M. Steinke ${ }^{9}$, G.Y. Sun ${ }^{1}$, A. Teufel ${ }^{2}$, W. Ullrich ${ }^{1}$, G.J. Wagner ${ }^{4}$, M. Wagner ${ }^{2}$, R. Wenzel ${ }^{1}$, A. Wilms ${ }^{9}$, P. Wintz ${ }^{3}$, S. Wirth ${ }^{2}$, P. Wüstner ${ }^{3}$, and P. Zupranski ${ }^{6}$

1 Institut für Kern- und Teilchenphysik, Technische Universität Dresden, D-01062 Dresden, Germany

2 Physikalisches Institut, Universität Erlangen-Nürnberg, D-91058 Erlangen, Germany

3 Institut für Kernphysik, Forschungszentrum Jülich, D-52425 Jülich, Germany

4 Physikalisches Institut, Universität Tübingen, D-72076 Tübingen, Germany

5 Institut für Strahlenphysik, Helmholtzzentrum Dresden-Rossendorf, Postfach 5101 19, D-01314 Dresden, Germany

6 Soltan Institute for Nuclear Studies, 05-400 Swierk/Otwock, Poland

7 INFN Torino, 10125 Torino, Italy

${ }^{8}$ Helmholtz Institut für Strahlen- und Kernphysik, Rheinische Friedrich-Wilhelm-Universität Bonn, D-53115 Bonn, Germany

9 Institut für Experimentalphysik, Ruhr-Universität Bochum, D-44780 Bochum, Germany

Received: 20 October 2011 / Revised: 16 January 2012

Published online: 28 February 2012

(c) The Author(s) 2012. This article is published with open access at Springerlink.com

Communicated by M. Guidal

\begin{abstract}
This paper reports results from a study of the reaction $\mathrm{pp} \rightarrow \mathrm{pK}^{0} \Sigma^{+}$at beam momenta of $p_{\text {beam }}=2950,3059$, and $3200 \mathrm{MeV} / c$ (excess energies of $\epsilon=126,161$, and $206 \mathrm{MeV}$ ). Total cross-sections were determined for all energies; a set of differential cross-sections (Dalitz plots; invariant-mass spectra of all two-body subsystems; angular distributions of all final-state particles; distributions in helicity and Jackson frames) are presented for $\epsilon=161 \mathrm{MeV}$. The total cross-sections are proportional to the volume of available three-body phase space indicating that the transition matrix element does not change significantly in this range of excess energies. It is concluded from the differential data that the reaction proceeds dominantly via the $N(1710) P_{11}$ - and/or $N(1720) P_{13}$-resonance(s); $N(1650) S_{11}$ and $\Delta(1600) P_{33}$ could also contribute.
\end{abstract}

\section{Introduction}

The study of associated strangeness production in protonproton collisions is one of the major physics programs carried out at the COoler SYnchrotron COSY located at Forschungszentrum Jülich, Germany. Various experimental groups have contributed data to the final states $p K^{+} \Lambda, p K^{+} \Sigma^{0}$, and $n K^{+} \Sigma^{+}$in the past decade [1-12]. In all these three cases the final states contain a rather long-lived charged kaon $\left(c \tau_{K^{+}}=3.7 \mathrm{~m}\right)$ and a nucleon, and thus are experimentally well suited to be accessed in inclusive and exclusive measurements at the COSY facility. In the case of $\Lambda$ and $\Sigma^{0}$ production this effort has led to excitation functions measured at excess energies $\left(\epsilon=\sqrt{s}-\left(m_{p}+m_{K}+m_{Y}\right)\right)$ from only a few $\mathrm{MeV}$

\footnotetext{
a e-mail: m.schulte-wissermann@physik.tu-dresden.de
}

above the threshold to $\epsilon \approx 250 \mathrm{MeV}$. Dalitz plots were investigated by the COSY-TOF Collaboration $[6,9]$ which, very recently, also published differential cross-section data for both reaction channels [10]. Along with the wealth of data, various theoretical approaches based on very different footings were proposed [13-19]. In the case of the $n K^{+} \Sigma^{+}$final-state total cross-sections were published for $\epsilon<200 \mathrm{MeV}[7,8,11,12]$ which, however, are contradicting each other strongly. This oddity is subject to an ongoing theoretical discussion [20].

Compared to the three final states discussed above the experimental data basis for the reaction $p p \rightarrow p K^{0} \Sigma^{+}$ is very scarce for $\epsilon \leq 350 \mathrm{MeV}$. In fact, the only experimental data for this reaction stem from the search for the supposed pentaquark state $\Theta^{+}\left(p p \rightarrow \Sigma^{+} \Theta^{+}, \Theta^{+} \rightarrow\right.$ $p K^{0}$ ) carried out by the COSY-TOF Collaboration [21]. 


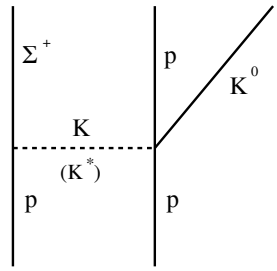

(a)

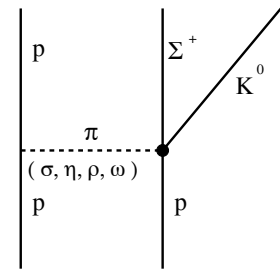

(b)

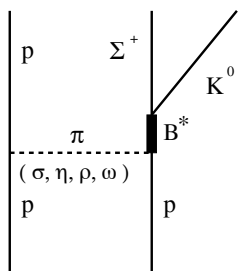

(c)
Fig. 1. Reaction mechanisms involving (a) strange, (b) nonresonant non-strange, and (c) resonant, non-strange meson exchange for $p p \rightarrow p K^{0} \Sigma^{+}$. Initial- and final-state interactions are not indicated.

At higher excess energies measurements of total crosssections were published originating from the bubble chamber experiments in the 1960s [22-29] (compiled in [30]). These data show, although with a large scatter, the total cross-section to be rather constant $(\approx 20 \mu \mathrm{b})$ over a wide energy range $(350 \mathrm{MeV}<\epsilon<2000 \mathrm{MeV})$. One of these early experiments reports a Dalitz plot [23] for $\epsilon=723 \mathrm{MeV}$. The approximately 30 entries show an enhanced density at small $K^{0} \Sigma^{+}$invariant masses which was interpreted as a hint for pion exchange.

Also from the theoretical point of view, the reaction $p p \rightarrow p K^{0} \Sigma^{+}$was only poorly addressed as compared to the reaction channels containing a $K^{+}$meson. In 1960, Ferrari [31] was the first to predict the total cross-section for excess energies between 72 and $400 \mathrm{MeV}$ in a meson exchange model where pion as well as kaon exchange were considered. A schematic diagram of the respective exchange graphs is shown in fig. 1 on the left for kaon exchange (strangeness manifests itself in the exchanged boson) and in the middle for pion exchange (associated strangeness production at the $p \pi \rightarrow K^{0} \Sigma^{+}$vertex). Another early calculation of the cross-sections simultaneously for all $p p \rightarrow N K Y$ channels was performed by Tsu Yao [32] for a beam energy of $T_{p}=2.85 \mathrm{GeV}$. This model is based solely on single-pion exchange and the calculated total cross-section for $p p \rightarrow p K^{0} \Sigma^{+}(\epsilon=353 \mathrm{MeV})$ is in rather good agreement with the prediction of Ferrari [31] and the only experimental value [22] known at that time. Later on, theoretical analyses and interpretations appeared mainly in experimental papers [23,24,26, 27]. In 1968, Ferrari and Serio [33] explained fairly well in a meson exchange model all the then known total and differential cross-sections for the $N K Y$ final state by introducing empirical cutoff factors in order to model various damping effects at large momentum transfer (form factors, absorption). Since then, the theoretical progress in describing the production of the $p K^{0} \Sigma^{+}$final state was strongly hampered by the lack of new experimental data. In view of the experimental and theoretical progress made for the $p K^{+} \Lambda$ and $p K^{+} \Sigma^{0}$ final states, in particular data at lower excess energies are highly desirable. This would render possible the further development of more recent theoretical approaches [34-36], which now also include, apart from kaon and pion exchange, a production scenario involving intermediate baryon resonances $\left(p p \rightarrow B^{*} p ; B^{*}=N^{*}, \Delta^{*} ; B^{*} \rightarrow K^{0} \Sigma^{+}\right.$, see fig. 1c).
If it is assumed that the associated strangeness production in pp-reactions procedes via resonances, a comprehensive study needs the consideration of $N^{*}$ - as well as $\Delta^{+*}$-resonances in the case of both $p p \rightarrow p K^{+} \Sigma^{0}$ and $p p \rightarrow p K^{0} \Sigma^{+}$reactions; due to isospin conservation the reaction $p p \rightarrow p K^{+} \Lambda$ can involve only $N^{*}$-resonances. The $p p \rightarrow n K^{+} \Sigma^{+}$-reaction, however, can proceed only via $\Delta^{++*}$-resonances.

In this paper we report results obtained for the reaction $p p \rightarrow p K^{0} \Sigma^{+}$measured for excess energies of $\epsilon=$ $126,161,206 \mathrm{MeV}\left(p_{\text {beam }}=2950,3059\right.$, and $\left.3200 \mathrm{MeV} / c\right)$. These data supplement earlier studies on the reaction channels $p p \rightarrow p p \omega \quad[37,38], p p \rightarrow p K^{+} \Lambda$, and $p p \rightarrow$ $p K^{+} \Sigma^{0}[10]$. As the data for these various reactions were taken simultaneously and the analyses utilized the same software package [39] the results obtained are characterized by a very high degree of internal consistency. This is, in particular, important for the future development of theoretic models when the three channels with associated strangeness production are to be described simultaneously.

For all three excess energies total cross-sections will be given. For the data taken at $\epsilon=161 \mathrm{MeV}$ Dalitz plots, invariant-mass spectra, distributions in the CMS, in helicity as well as in Jackson frames will be presented. All differential distributions are scrutinized with the aim of gaining insight into the reaction mechanism. It will be presented that all differential distributions can be described simultaneously and consistently if a resonant production of the final state is assumed.

\section{Experimental procedure}

\subsection{Detector setup}

The experiments were carried out with the time-offlight detector COSY-TOF located at an external beam line of the COoler SYnchrotron COSY (Forschungszentrum Jülich). The COSY machine provides proton beams of very high quality (spill length $\approx 5 \mathrm{~min}$; several $10^{6}$ protons/s; low emittance of $<5 \pi \mathrm{mm}$ mrad; relative momentum uncertainty $\Delta p / p<10^{-3}$ ).

The layout of the COSY-TOF detector is shown in the upper part of fig. 2; in the lower part the near target region with the time-of-flight start and tracking detectors $[40,41]$ is sketched. The interaction volume is small and well defined as the narrow beam with Gaussian profile $\left(\sigma_{x, y}<300 \mu \mathrm{m}\right)$ is directed onto a liquid-hydrogen target of only $4 \mathrm{~mm}$ length [42]. The emerging particles traverse just behind the target $(\approx 25 \mathrm{~mm})$ a 24 -fold segmented scintillation detector ("start-detector") which provides the start signal for the time-of-flight measurement. At a distance of $30 \mathrm{~mm}$ downstream of the target a doublesided silicon-microstrip detector is installed, followed by two double-layered scintillating fiber hodoscopes at 100 and $200 \mathrm{~mm}$. These three tracking detectors measure the coordinates of traversing charged particles in three dimensions with a spatial resolution of $\approx 100 \mu \mathrm{m}$ (microstrip) and $\approx 1.5 \mathrm{~mm}$ (hodoscopes). 

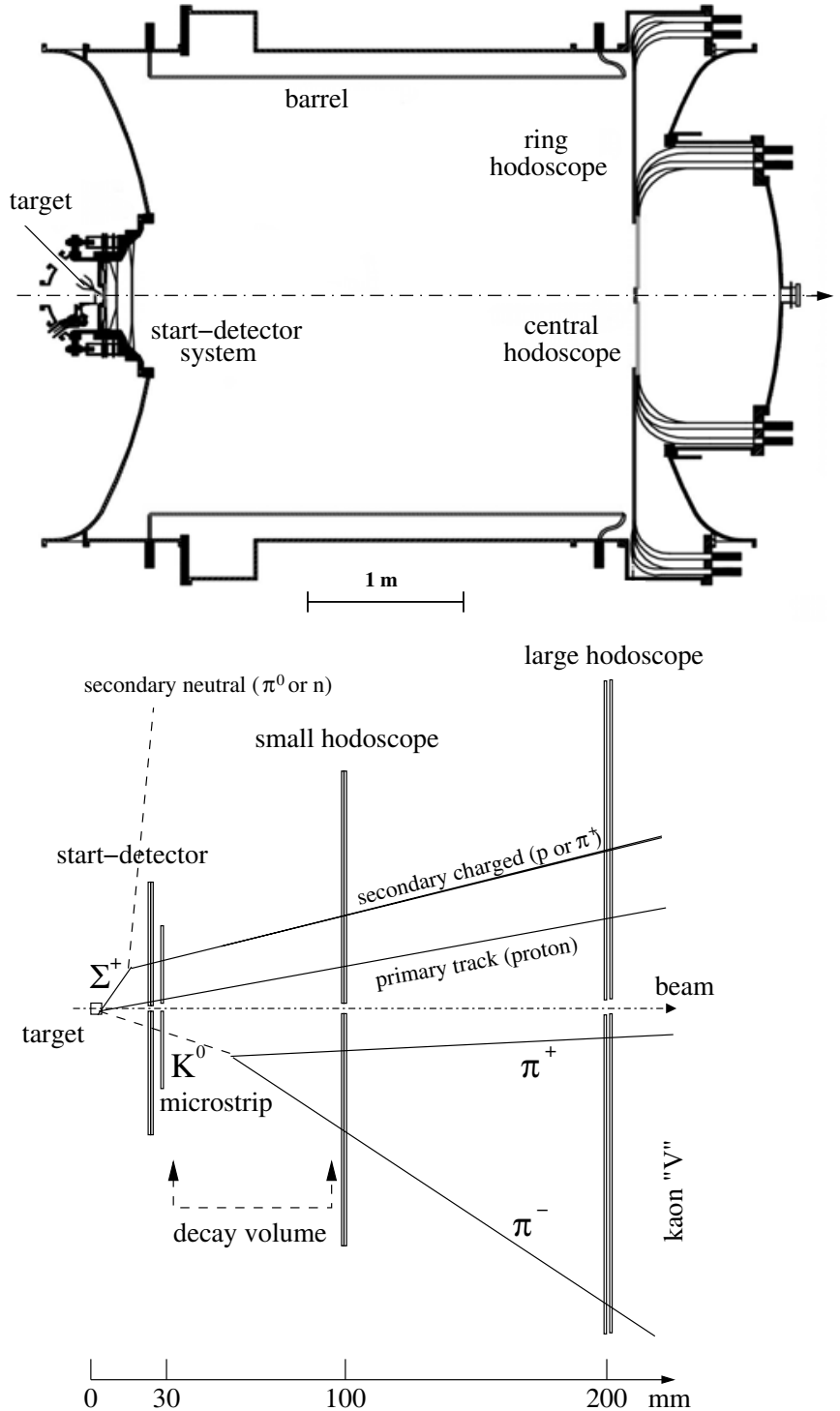

Fig. 2. The COSY-TOF detector (top), the near-target region (start-detector system, bottom). The lower picture shows a typical event pattern for the $p K^{0} \Sigma^{+}$exit channel.

After a flight path of $\approx 3 \mathrm{~m}$ through the evacuated vessel $(0.2 \mathrm{~Pa})$ all charged particles are detected in the highly segmented stop components. They consist of two triple-layered forward hodoscopes (central and ring hodoscope) [43] and the barrel hodoscope [44], all manufactured from BC412 scintillating material. From the combined measurement of time and position the velocity vectors of charged particles (originating from the target) are determined with a time-of-flight resolution of better than $\sigma_{T O F}=300 \mathrm{ps}$ and an angular track-resolution of better than $\sigma_{\varangle}=0.3^{\circ}$. Vertices from neutral particles decaying behind the microstrip detector and in front of the first hodoscope are reconstructed from the tracks of their two charged daughter particles with an accuracy of $\sigma_{x, y}<1 \mathrm{~mm}$ and $\sigma_{z}<3.0 \mathrm{~mm}$. For the present analysis this feature is of crucial importance as a measurement of the four-momentum of the $K_{S}^{0}$ is mandatory for the full kinematic reconstruction of the final state.
The COSY-TOF detector stands out for its low mass areal density of target, start-detector, and tracking detectors. This renders the influence of small angle scattering and energy loss almost negligible. In addition, the COSYTOF detector has a high efficiency of $>95 \%$ for the detection of charged particles and covers a large solid angle $\left(1^{\circ}<\theta<60^{\circ}, 0^{\circ}<\phi<360^{\circ}\right)$ in the laboratory frame. These features allow the study of different reaction channels, (e.g., $p p \rightarrow p p$ [45], $p p \gamma$ [46], $p p \eta[47], d \pi^{+}$[39], $p p \omega[38], p K^{0} \Sigma^{+}[21]$, and $p K^{+} \Lambda$ as well as $\left.p K^{+} \Sigma^{0}[10]\right)$ from the same data sample by examining the measured time-of-flight of the charged particles and the overall event topology.

\subsection{Principle of measurement and data analysis}

The TOF start-detector setup was designed to provide an effective means for the analysis of final states with open strangeness (e.g., $p K^{+} \Lambda, p K^{+} \Sigma^{0}, p K^{0} \Sigma^{+}$). Here, two charged particles are emitted at the three-particle production vertex. In the case of $p p \rightarrow p K^{0} \Sigma^{+}$the $K_{L}^{0}$ escapes the detector $\left(\mathrm{c} \tau_{K_{L}^{0}}=15.3 \mathrm{~m}\right)$ while the $K_{S}^{0}$ has a considerable probability $\left(\mathrm{c} \tau_{K_{S}^{0}}=26.8 \mathrm{~mm}\right)$ to decay into two charged particles behind the start-detector and in front of the first hodoscope (see fig. 2, lower part). Hence, the start-detector will be hit by only two, while the detectors located further downstream, i.e. the two hodoscopes and the stop detectors, will be hit by four charged particles. This "multiplicity jump of charged particles" is set as a trigger condition during data taking and is also the first condition required in the off-line analysis.

In order to discriminate the reaction $p p \rightarrow p K^{0} \Sigma^{+}$ from background, the characteristics of its final states is exploited: 1) a prompt track emerging from the target (proton), 2) a decay "V" $\left(K_{s}^{0} \rightarrow \pi^{+} \pi^{-}\right)$with its vertex located in the decay volume (displaced vertex), and 3 ) one additional hit somewhere in the stop detector (due to the charged decay particle from either $\Sigma^{+} \rightarrow p \pi^{0}$ or $\Sigma^{+} \rightarrow$ $\left.n \pi^{+}\right)$.

Primary track and decay $\mathrm{V}$ candidates are selected by applying the following conditions: a primary track must have a signal in the start-detector and in one of the stop components. In addition, at least two signals from the three subdetectors (microstrip detector, small and large hodoscope) are required. A secondary $\mathrm{V}$ must consist of two independent arms, each defined by fitting points in both hodoscopes and the stop detector. The point of closest approach of both arms is considered to be the kaon decay vertex; the vector connecting the center of the target and this decay vertex is considered to be the kaon's direction of flight $\left(\hat{p}_{K_{S}^{0}}\right)$. In order to discriminate a secondary $\mathrm{V}$ candidate against the background generated by primary particles inducing reactions in the start-detector a minimum angle of the pion with respect to the mother particle of $10^{\circ}$ and a minimum angle between both pions of $30^{\circ}$ is required.

All permutations of primary tracks and decay $\mathrm{V}$ candidates are subjected to an overall quality check which 
includes the number of involved fitting points, the distance of closest approach of both tracks of the decay $\mathrm{V}$, the quality of all fittings procedures ( $\chi^{2}$ values), and the coplanarity of the decay $\mathrm{V}$ with respect to the primary kaon $\left(\hat{p}_{K_{S}^{0}} \times\left(\hat{p}_{\pi^{+}} \times \hat{p}_{\pi^{-}}\right) \approx 0\right)$. The combination of a primary track and a secondary $\mathrm{V}$ with the best overall quality is kept for further analyses. This method was developed by means of Monte Carlo data which show that in the final event sample $86 \%$ of the events are reconstructed correctly.

So far only geometric information is exploited in order to identify the primary proton and the two decay pions of the primary (neutral) kaon. The velocity vector of a primary track is calculated from $\left(t^{\text {stop }}-t^{\text {start }}\right)$ and by assigning the proton mass to this velocity vector the proton four-momentum is obtained.

As the secondary pions from the $K_{S}^{0}$ decay emerge behind the start detector they lack an individual start-time information. Nevertheless, their momenta can be calculated from the kinematic relations between the measured angles of the daughter pions with respect to their mother kaon. The sum of both pion four-momenta then yields the four-momentum of the kaon. The four-momenta of beam, target, kaon, and proton are used in order to calculate the four-momentum of the $\Sigma^{+}\left(\mathcal{P}_{\Sigma^{+}}=\mathcal{P}_{b}+\mathcal{P}_{t}-\mathcal{P}_{K^{0}}-\mathcal{P}_{p}\right)$ The invariant mass of the $\Sigma^{+}$is the "missing mass" of the final state. The resulting missing-mass spectrum is the backbone of the analysis.

Although the two pions do not provide an individual start-time signal their stop-time information can nevertheless be used as a means to substantially reduce experimental background [48]. For this purpose the difference of the time of flights of the two pions is calculated via

$$
\begin{aligned}
\Delta_{\text {tof }} & =\left(t_{\pi_{1}}^{\text {stop }}-t_{\pi_{1}}^{\text {start }}\right)-\left(t_{\pi_{2}}^{\text {stop }}-t_{\pi_{2}}^{\text {start }}\right) \\
& =t_{\pi_{1}}^{\text {stop }}-t_{\pi_{2}}^{\text {stop }}
\end{aligned}
$$

as both pions are created simultaneously $\left(t_{\pi_{1}}^{\text {start }}=t_{\pi_{2}}^{\text {start }}\right)$. This measured time difference is then compared to the time difference determined from the path length of the pions in the detector and the pions' calculated momenta. Measured and calculated time differences must match within $2 \mathrm{~ns}\left(\left|\Delta_{\text {tof }}^{\text {calc }}-\Delta_{\text {tof }}^{\text {meas }}\right| \leq 2 \mathrm{~ns}\right)$. This requirement does not change the number of identified events significantly in the final event sample. The amount of experimental background, however, is reduced by about a factor of two.

It should be noted that the information of the $\Sigma^{+}$ enters only by the presence of a fourth charged particle somewhere in the detector. The characteristic "kinkangle" (cf. lower part of fig. 2) could be used in order to discriminate between the two main decay channels of the hyperon $\Sigma^{+} \rightarrow p \pi^{0}$ and $\Sigma^{+} \rightarrow n \pi^{+}$. In fact, due to the mass difference of the charged daughter particles $90 \%$ of the $p \pi^{0}\left(n \pi^{+}\right)$decay branch is found with a kink-angle below (above) $12^{\circ}$. The ratio of the acceptance corrected counts is $N_{\Sigma^{+} \rightarrow p \pi^{0}} / N_{\Sigma^{+} \rightarrow n \pi^{+}}=0.99 \pm 0.16$, i.e. fully compatible with the accepted ratio of the branching ratios $\left(\mathcal{B}_{\Sigma^{+} \rightarrow p \pi^{0}} / \mathcal{B}_{\Sigma^{+} \rightarrow n \pi^{+}}=1.07[49]\right)$. However, no selection based on the kink-angle has been applied in the final analysis since it neither improves the missing-mass resolution nor increases the signal-to-background ratio substantially.

The effects of various background reactions on the final missing-mass spectrum were studied by Monte Carlo simulations. For this purpose $10^{7}$ events, distributed according to equal population of the available phase space, were generated for each of the reactions $p p \rightarrow p p, d \pi^{+}$, $p p \pi^{0}, p n \pi^{+}, p p \omega, p K^{+} \Sigma^{0}$ and $p K^{+} \Lambda$ and subjected to the $p p \rightarrow p K^{0} \Sigma^{+}$analysis routines. All but the hyperon channels produce negligible $\left(<1 / 10^{6}\right)$ or no background at all. The reactions $p p \rightarrow p K^{+} \Sigma^{0}$ and $p p \rightarrow p K^{+} \Lambda$ cause a broad missing mass spectrum; no peak at the $\Sigma^{+}$mass is observed in either cases. The acceptance for both channels is about $1.5 \%$ with respect to the $p K^{0} \Sigma^{+}$channel. Considering the total cross-sections for the $\Lambda$ and $\Sigma^{0}$ channels [10] their added contribution to the final event sample is below $15 \%$ for each of the three measured beam momenta.

\subsection{Acceptance correction and absolute normalization}

The Monte Carlo package used [50,51] models the detector and the physical processes to great detail. The event generator produces the particles of the exit channel either according to three-body phase space, or alternatively, intermediate resonances can be chosen in order to model a two-step production process $\left(p p \rightarrow p N^{*}, N^{*} \rightarrow K^{0} \Sigma^{+}\right)$. The particles (and their daughters, granddaughters, etc.) are then propagated through the detector. Branching ratios and lifetimes of all particles are incorporated according to the values given in [49]. Energy loss, smallangle scattering, nuclear reactions, and $\delta$-electrons are considered. Digitized QDC- and TDC-signals are generated from the energy deposited in the active detector components. Noise and thresholds are modelled as known from the measured detector response. Deviations from an homogeneously populated phase space can be introduced by weight functions on an event-by-event basis (this procedure is called filtering in the following). Finally, the Monte Carlo data are subjected to the same routines as real data in order to determine the acceptance ${ }^{1}$.

The overall acceptance of about one percent for the reaction under study is determined by the ratio of positively identified events to the number of generated $\left(10^{7}\right)$ Monte Carlo events. This value is well explained by the event kinematics, the detector geometry, and the detector performance: the probability to observe the neutral kaon via its $K_{S}^{0}$ component $(50 \%)$, the branching ratio of the kaon to two charged pions $(69.2 \%)$, the probability of the secondary vertex to be located within the fiducial "decay volume" (31\%), the probability of both secondary pions to traverse the active detector range $(23 \%)$, the reconstruction efficiency for both pions (60\%), the reconstruction efficiency of a primary proton track (95\%),

\footnotetext{
1 Throughout this paper, the term "acceptance" is used for the convolution of solid angle coverage, detector, and reconstruction efficiency.
} 

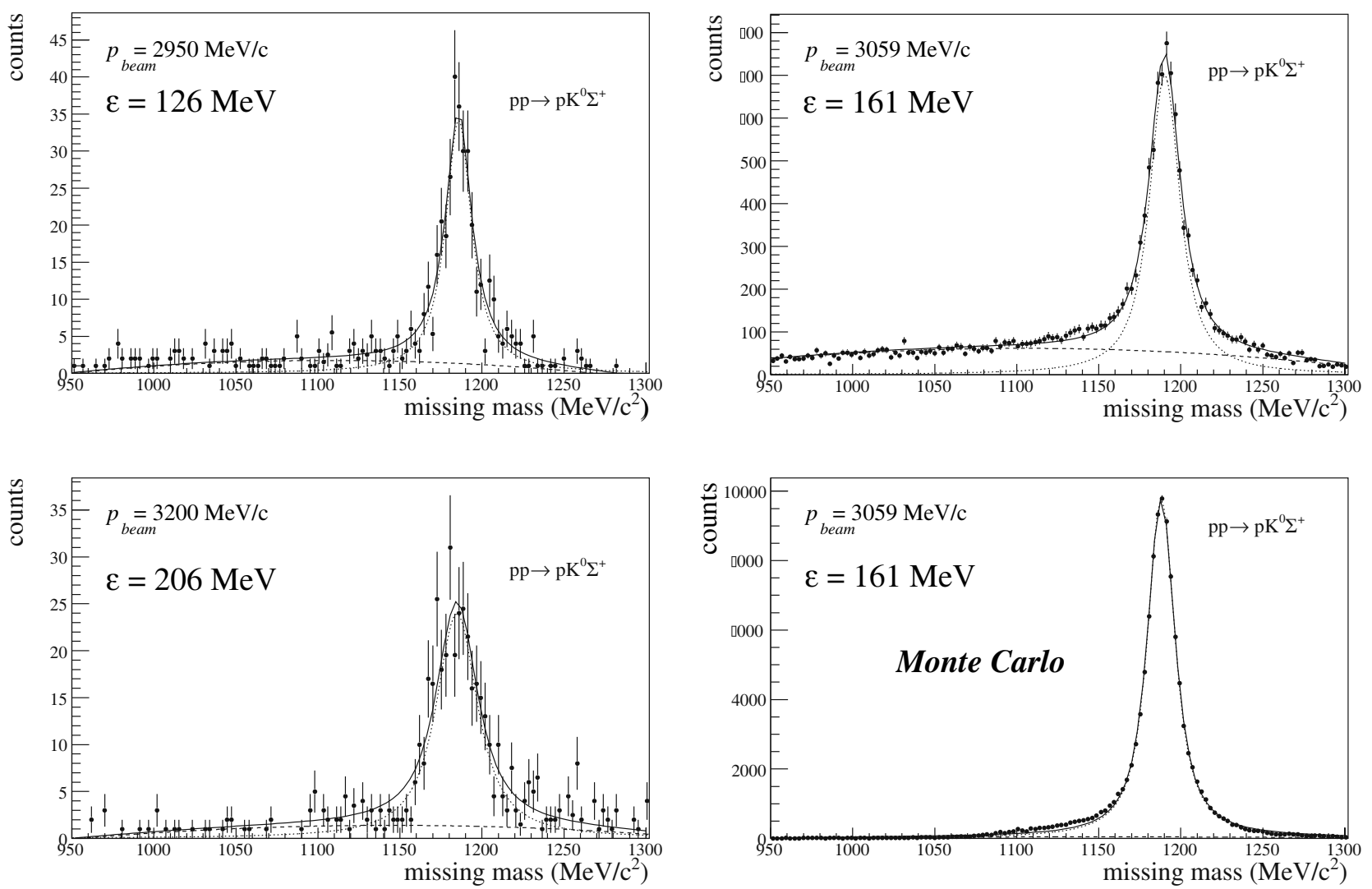

Fig. 3. Proton- $K^{0}$ missing-mass spectra measured for the three excess energies. A prominent peak is observed above a smooth background in all cases. The results of a fitting procedure for signal (dotted line), background (dashed line), and total spectrum (solid line) are shown in all cases. In the lower right frame the Monte Carlo result for $\epsilon=161 \mathrm{MeV}$ is depicted.

and the detection probablilty of the decay particle of the hyperon $(82 \%)$. The acceptance has been subject to a detailed reevaluation, resulting in a modification of the total cross-section at $\epsilon=161 \mathrm{MeV}$ presented below compared to the previously published value [21].

The relative uncertainty of the overall acceptance correction was determined by considering the following effects: 1) The efficiency for the detection of charged particles is generally high $(>95 \%)$ and known with an uncertainty of $\pm 5 \%$ for each of the $\approx 1500$ individual detector channels. This leads to a contribution to the uncertainty of the acceptance correction of $14 \%$ for the present reaction. 2) By altering all restrictions imposed during the data analysis the number of $p K_{s}^{0} \Sigma^{+}$events in the final event sample can be changed by $+10 \% /-70 \%$ while the total cross-section changes by less than $10 \%$. This value is taken as the contribution of the choice of the restrictions used in the analysis to the overall uncertainty of the acceptance correction. 3) The influence of the choice of intermediate nucleon resonances (MC input) and angular distributions (filter) was found to be small ( $2 \%$ each), if the mass and width of the resonance and the asymmetry of the filter functions are altered within the limits imposed by the measurements (see below). Adding these contributions quadratically an overall systematic uncertainty of $18 \%$ is obtained.
With regard to differential distributions the acceptance varies in all cases smoothly with the observable under consideration (details will be shown later when presenting the final results). Here, an additional uncertainty $\Delta a_{i}$ comes about for each bin $i$ due to the gradient of the acceptance function $a_{i}$. This is accounted for by choosing as uncertainty the mean change of $a_{i}$ with regard to its adjacent bins, i.e. $\Delta a_{i}=\frac{1}{2}\left(\left|a_{i}-a_{i-1}\right| / 2+\left|a_{i}-a_{i+1}\right| / 2\right)$.

The absolute normalization is determined via the analysis of elastic scattering, which was recorded simultaneously during the experiment. Our results are normalized to the elastic scattering cross-sections from the EDDA collaboration [52] and yielded time-integrated luminosities of $16.9 \mathrm{nb}^{-1}(\epsilon=126 \mathrm{MeV}), 214 \mathrm{nb}^{-1}(161 \mathrm{MeV})$, and $6.4 \mathrm{nb}^{-1}(206 \mathrm{MeV})$. The total uncertainty of this procedure $(5 \%)$ is due in equal parts to our analysis and the uncertainty of the literature data. For details see $[39,45]$.

\subsection{Determination of total and differential cross-sections}

Figure 3 shows the $p K^{0}$ missing-mass spectra obtained for the three excess energies of 127, 161, and $206 \mathrm{MeV}$. Prominent signals for the $\Sigma^{+}$hyperon can be seen at its central mass of $1189 \mathrm{MeV} / c^{2}$ above a smooth and structure- 
less background. As usual for time-of-flight detectors, the missing-mass resolution (momentum resolution) is best for smaller velocities in the exit channel (smaller beam momenta in the entrance channel). When comparing the spectra one clearly notes the higher integrated luminosity for $\epsilon=161$. At $\epsilon=126$ and $206 \mathrm{MeV}$, however, a better beam quality was available, as manifested by the lower background contribution. In the lower right frame the Monte Carlo result is shown for $\epsilon=161 \mathrm{MeV}$.

The number of events in the missing-mass peak are obtained by consecutively fitting first the background and then the signals. The background is parameterized by a quadratic polynomial, where only missing masses below and above the $\Sigma^{+}$-peak are taken into account $\left(m<1100 \mathrm{MeV} / c^{2}\right.$ and $\left.m>1250 \mathrm{MeV} / c^{2}\right)$. Then, the background parameters are fixed and the signal is described by a Voigt function (convolution of a Gauss and a Lorentz function). Voigt functions are chosen since they model properly the signal shape of a rather narrow peak accompanied by broader tails. The integral of the Voigt function represents the total number of positively identified events. The overall systematic uncertainty due to signal and background separation is determined by varying the fit region for the background fit below and above the $\Sigma^{+}$-peak and was found to be $\pm 4 \%$.

The total cross-sections for all three excess energies are then obtained from the number of identified events $(N)$, the integrated luminosity $(\mathcal{L})$, and the acceptance (a) according to $\sigma=N /(\mathcal{L} \cdot a)$. As statistical uncertainties the numerical uncertainties of the fitting procedure of the signal will be quoted. As systematic uncertainties the quadratic sum of the uncertainties of luminosity determination (5\%), acceptance correction (18\%), and signal-tobackground separation (4\%) will be given.

Differential cross-sections are determined in analogy to the total yield, only that the amount of signal and background is determined from individual missing-mass spectra generated for each bin of the observable under study. Also the uncertainty of the signal-background separation is determinded individually. Data will be shown if the uncertainty in a specific bin (root mean square of the statistical uncertainty combined with the differential uncertainty of the acceptance correction and the signalbackground separation) is below $80 \%$ of its cross-section value.

The numerical values of all total and differential crosssections will be given below. For the latter case the coefficients of least square fitting with Legendre polynomials

$$
\mathrm{d} \sigma / \mathrm{d} \Omega=\sum_{l=0}^{l_{\max }} a_{l} \cdot P_{l}, \quad l=0,1,2
$$

will be given in order to judge asymmetries $\left(P_{1}\right.$, representative for all $\left.P_{o d d}\right)$ and anisotropies $\left(P_{2}\right)$. It should be noted in passing that the integrals of all angular distributions $\left(\sigma_{t o t}=\int \frac{\mathrm{d} \sigma}{\mathrm{d} \Omega} \mathrm{d} \Omega=4 \pi \cdot a_{0}\right)$ match the total crosssection within uncertainty.
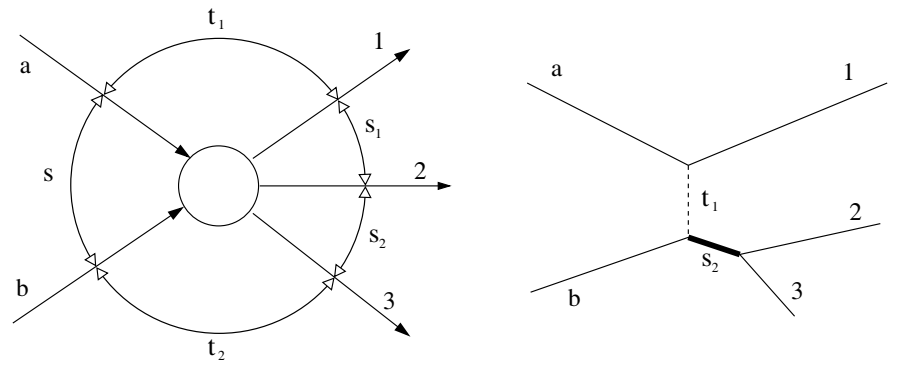

Fig. 4. Graphical representation of $s, s_{1}, s_{2}, t_{1}, t_{2}$ following the prescription of ref. [53] for the most general case of a $a b \rightarrow 123$ reaction (left), and for the case of a two-step reaction involving an intermediate resonance (right).

\section{Results and discussion}

For a reaction of type $a+b \rightarrow 1+2+3$ the reaction space given by kinematics is 12-dimensional (three 4-momentum vectors of the exit channel), if the entrance channel is fixed (masses, $\sqrt{s}$ ). Knowing the masses of the exit channel reduces the dimensionality to 9 (three 3-momentum vectors). Four energy-momentum conservation equations reduce the dimensionality to five. If no spin direction is preferred, the azimuthal dependence is trivial and four dimensions (variables) remain in order to uniquely describe the reaction kinematics.

The actual choice of the linearly independent basis of such a four-dimensional space (or four experimental observables) is not unique. One convenient choice are independent Mandelstam-like invariants [53]:

$$
\begin{aligned}
& s_{1} \equiv s_{12}=\left(\mathcal{P}_{1}+\mathcal{P}_{2}\right)^{2} \\
& s_{2} \equiv s_{23}=\left(\mathcal{P}_{2}+\mathcal{P}_{3}\right)^{2} \\
& t_{1} \equiv t_{a 1}=\left(\mathcal{P}_{a}-\mathcal{P}_{1}\right)^{2} \\
& t_{2} \equiv t_{b 3}=\left(\mathcal{P}_{b}-\mathcal{P}_{3}\right)^{2}
\end{aligned}
$$

where the $\mathcal{P}_{i}$ denote the four-momentum of particle $i$. The physical meaning of these variables can be infered from fig. $4: t_{1}, t_{2}$ are the squared momentum transfers between the initial particles and two of the three ejectiles; $s_{1}, s_{2}$ are the squared invariant masses of two of the three final state subsystems. In case of resonant production we adopt the convention that $t_{1}$ is the momentum carried by the exchange particle while $t_{2}$ is the momentum transfer from particle $b$ to one of the decay products of the resonance. In the case of a symmetric entrance channel as in the current case particles $a$ and $b$ are interchangeable.

Using these four variables the four-fold differential cross-section can be written as

$$
\mathrm{d}^{4} \sigma / \mathrm{d} s_{1} \mathrm{~d} s_{2} \mathrm{~d} t_{1} \mathrm{~d} t_{2}=\Phi \cdot\left|M\left(s ; s_{1}, s_{2}, t_{1}, t_{2}\right)\right|^{2},
$$

where $\Phi$ represents the properly normalized phase-space factor and $M$ is the transition matrix element.

The four relativistic invariants in eq. (4) are linearly connected to specific angles, for instance, $s_{1}$ is a linear function of $\cos \left(\angle\left(\boldsymbol{p}_{\mathbf{1}}, \boldsymbol{p}_{\mathbf{2}}\right)\right)$ in a properly chosen reference frame. Similarly, other angles can be used to substitute $s_{2}$, 
Table 1. Total cross-sections for the reactions $p p \rightarrow p K^{0} \Sigma^{+}$ for the three different excess energies. The first uncertainty refers to statistical and the second to systematical ones.

\begin{tabular}{cccc}
\hline$\varepsilon(\mathrm{MeV})$ & Acceptance $(\%)$ & Signal $(\#)$ & $\sigma_{\text {tot }}(\mu \mathrm{b})$ \\
\hline 127 & 0.93 & 386 & $2.46 \pm 0.13 \pm 0.47$ \\
161 & 1.05 & 9226 & $4.13 \pm 0.06 \pm 0.79$ \\
206 & 0.93 & 412 & $7.02 \pm 0.36 \pm 1.34$ \\
\hline
\end{tabular}

$t_{1}, t_{2}$. Therefore, the four-fold differential cross-section can also be written as a function of a combinations of angles and invariants.

The experimental goal is to provide the four-fold differential cross-section (in whatever basis). This, however, is unrealistic in many cases, simply for statistical reasons. In practice, one therefore needs to reduce the dimensionality by projecting onto subspaces: Integration over $t_{1}$ and $t_{2}$ results in Dalitz plots. Projecting onto one dimension yields, for instance, invariant masses or angular distributions in particular reference frames.

The reduction of dimensionality is accompanied with a significant loss of information, as possible correlations can no longer be recognized. In addition, one has to be cautious about the kinematic correlation of the three-body final state, as in specific cases a true physical cause in one variable can mimic a characteristic signal in another variable [54] (kinematic reflection). It therefore is essential to evaluate as many as possible (linear independent) projections of the four-fold differential cross-section. These projections are then to be described simultaneously by a theoretical model.

\subsection{Total cross-sections}

Integration over all four variables in eq. (4) results in the total cross-section. Thus, a single value for the total crosssection does not allow to infer any details of the reaction mechanism. Nevertheless, the evolution of the total cross-section with excess energy, the excitation function $(\sigma=\sigma(\sqrt{s}))$, is often used as a first means to judge how well (different) theoretical approaches are appropriate to describe these data.

The results for the total cross-section are listed in table 1 . They are included in fig. 5 which shows the world data for the reaction $p p \rightarrow p K^{0} \Sigma^{+}$. All data above $\epsilon=300 \mathrm{MeV}$ stem from bubble chamber experiments [2230]. The data show a relatively large scatter, however indicate a rather constant total cross-section over a wide energy range. The present data are the first to establish the excitation function at smaller excess energies.

The results of two early theoretical approaches are shown in the same figure. In 1962 Tsu Yao [32] calculated the total cross-section for the present reaction at $\epsilon=353 \mathrm{MeV}$ considering solely single-pion exchange. The resulting value of $\sigma=36 \mu \mathrm{b}$ is in good agreement with the only experimental value [22] available at that time. The calculation by Ferrari and Serio in 1968 [33] (based on the

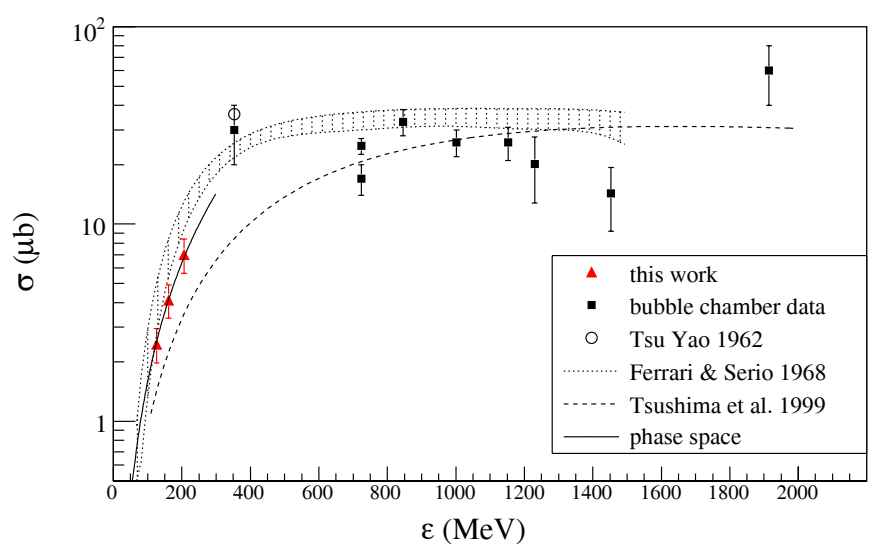

Fig. 5. Total cross-sections of the reaction $p p \rightarrow p K^{0} \Sigma^{+}$. The triangles at low excess energies represent the present data. The solid square symbols are from bubble chamber experiments (compiled in [30]). The theoretical calculations shown are described in the text.

pioneering work of Ferrari [31]) was aimed at the simultaneous description of various $p p \rightarrow N K Y$ channels within a boson exchange model (explicitly excluding nucleon resonances). The energy dependence of the total cross-section calculated by this model is shown as dotted lines for excess energies up to $1500 \mathrm{MeV}$. Here, the lower line represents the results for purely pion exchange, while the additional contribution of kaon exchange (for a coupling constant of $G_{\Sigma}^{2}$ less than or equal to 1.6) is represented by the hatched area. The calculation for pure pion exchange is in rather good agreement with our data. Nevertheless, it will be shown in the following that nucleon resonances play an important role for the production of the $p K^{0} \Sigma^{+}$final state. Hence, the model of Ferrari and Serio is lacking a central ingredient and the good agreement between the experimental data and theoretical prediction is probably a coincidence.

More recent calculations by Tsushima et al. [36] are based on a resonance model where it is assumed that an excited baryon, $B^{*}\left(N^{*}\right.$ or $\left.\Delta^{*}\right)$, is produced via meson $(\pi, \eta, \rho)$ exchange $\left(p p \rightarrow p B^{*} ; B^{*} \rightarrow K^{+} \Lambda, K^{+} \Sigma^{0}\right.$, $K^{0} \Sigma^{+}$). With one set of parameters (coupling constants, cut-off values) all these three hyperon channels are treated on equal footing. In the case of $p p \rightarrow p K^{0} \Sigma^{+}$this model describes the total cross-sections measured at excess energies above $800 \mathrm{MeV}$ rather well (dashed curve), however misses the experimental value at $\epsilon=353 \mathrm{MeV}$ by about a factor four. This model underestimates the new data by a factor two.

We also include a solid line which represents the volume of available phase space $\left(\sigma \propto a \cdot \epsilon^{2}, a=1.617\right.$. $\left.10^{-4} \mu \mathrm{b} \mathrm{MeV}^{-1}\right)$. It describes the data very well within the given experimental uncertainty. This indicates that the absolute value of the transition matrix element in eq. (4) does not depend strongly on excess energy in the investigated energy region. However, this does not imply that the transition matrix element itself is constant over the available phase space. 

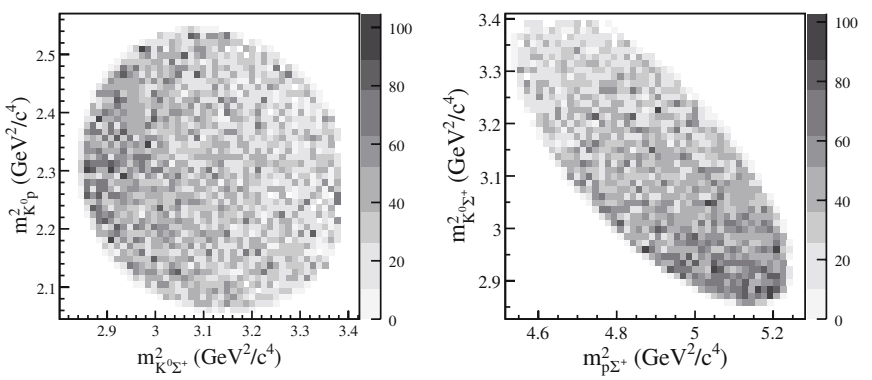

Fig. 6. Dalitz plots of the squared invariant masses of the $K^{0} p$ vs. $K^{0} \Sigma^{+}$(left) and $K^{0} \Sigma^{+}$vs. $p \Sigma^{+}$(right) subsystems for $\epsilon=161 \mathrm{MeV}$. The data is acceptance corrected, however the background is not subtracted (see text). In both frames a clear enhancement at low $K^{0} \Sigma^{+}$masses indicate the presence of an intermediate resonance state $\left(B^{*} \rightarrow K^{0} \Sigma^{+}\right)$located near the lower mass boundary. A contribution of a $p \Sigma^{+}$FSI would lead to an enhancement of data at the lower mass boundary of the $p \Sigma^{+}$mass axis. A significant signal of $p \Sigma^{+}$FSI is not observed.

\subsection{Dalitz plot}

Integrating over $t_{1}$ and $t_{2}$ in eq. (4) results in the doubledifferential cross-section $\mathrm{d}^{2} \sigma / \mathrm{d} s_{1} \mathrm{~d} s_{2}$, whose representation is the Dalitz plot [55]. It connects two Lorentz invariants (e.g., $s_{1}=m_{12}^{2}, s_{2}=m_{23}^{2}$, squared invariant masses) and, hence, gives insight into the correlation of the three particle final state. If the matrix element in eq. (4) is constant, i.e. does not depend on $s_{1}, s_{2}, t_{1}, t_{2}$, the Dalitz plot is homogeneously populated. If, however, the matrix element is not constant, the deviation from a uniformly populated phase space may show up as characteristic distortions. Prominent examples are resonances and FSI effects. If these signals are well separated, direct conclusions on physical properties can be drawn (e.g. mass and width of a resonance; scattering lengths via FSI). This simple interpretation is not possible if different effects overlap on the Dalitz plot. In this case a proper theoretical model (treating the dependence of the matrix element on all four variables $\left.s_{1}, s_{2}, t_{1}, t_{2}\right)$ must consider the different contributions coherently on the amplitude level.

Acceptance-corrected Dalitz plots $\left(m_{\mathrm{K}^{0} \mathrm{p}}^{2}\right.$ vs. $m_{\mathrm{K}^{0} \Sigma^{+}}^{2}$ and $m_{\mathrm{K}^{0} \Sigma^{+}}^{2}$ vs. $\left.m_{\mathrm{p} \Sigma^{+}}^{2}\right)$ are shown in fig. 6 for an excess energy of $161 \mathrm{MeV}$. Data is shown for a missing-mass region of $\pm 40 \mathrm{MeV} / c^{2}$ around the $\Sigma^{+}$mass, thereby reducing the contribution of background to below $20 \%$. The subtraction of background individually for each bin is not feasible for statistical reasons. However, sideband cuts show that the structures do not originate from the background.

In both Dalitz plots structures due to narrow resonances or final-state interaction are not observed within resolution. However, the relative bin occupancy of both Dalitz plots continuously increases towards low $K^{0} \Sigma^{+}$ masses. This can be interpreted to be caused by one or more intermediate resonances $\left(B^{*} \rightarrow K^{0} \Sigma^{+}\right)$with central masses $\left(m_{i}\right)$ in the region of the lower mass boundary (or below) and broad widths $\left(\Gamma_{i}\right)$ of at least $100 \mathrm{MeV}$. The
PDG [49] offers a whole list of baryon resonances which could potentially interfere and contribute to the reaction. Due to the arguments given above, it is not trivial to extract the resonance parameters $\left(m_{i}, \Gamma_{i}\right)$ from the Dalitz plots alone. In the following, however, we are going to argue that for the specific case of $p p \rightarrow p K^{0} \Sigma^{+}$a set of one dimensional distributions is well suited to shed light on the reaction mechanism, particularly on the resonant contribution.

\subsection{Angular distributions in the helicity frames and invariant-mass distributions}

Invariant-mass distributions are obtained by projecting the Dalitz plot onto one of its axes, $s_{1}=m_{12}^{2}, s_{2}=m_{23}^{2}$, or $s_{3}=m_{31}^{2}$. Although the resonance parameters $(m, \Gamma)$ are reflected in the invariant-mass spectra, their direct extraction is only possible for an isolated non-interfering resonance. In any case, one has to be cautious about kinematic reflections as the three invariants are related via $s_{1}+s_{2}+s_{3}=s+m_{1}^{2}+m_{2}^{2}+m_{3}^{2}$. Any structure deviating from phase space in, e.g., $s_{2}$, will cause deviations of the pure phase-space behavior of the other observables $s_{1}$ and $s_{3}$. It is therefore not a priori clear which invariant shows a cause and which an effect. In contrast, the situation is more clear when analyzing the angular distributions in helicity frames.

Angular distributions in helicity frames ${ }^{2}$ are essentially special projections of a Dalitz plot. There is a linear relation between $s_{i}$ and $\cos \theta_{i j}^{R j k}$ (and cyclic permutations), therefore $s_{1}$ and $s_{2}$ can be substituted by two helicity angles in eq. (4) [53].

For a single resonance (in the following we assume it to decay into the 23-system) the properties of mass and width lead to anisotropic distributions in the 12 - and $13-$ helicity frames, while the angular distribution in the 23helicity frame remains isotropic (projection of the "resonance band" in the Dalitz plot). This isotropy in the 23helicity frame is independent of mass and width of the resonance and therefore uniquely identifies the decay channel. This statement is also valid if more than one resonance (decaying into the same two-body system) contribute incoherently.

The angular distributions in all three helicity frames measured at an excess energy of $\epsilon=161 \mathrm{MeV}$ are shown in the upper row of fig. 7 . In the lower row the spectra of all three invariant-mass subsystems are displayed. The acceptances shown under each distribution are rather constant for all spectra. Due to the the method of determining the yield the physical background is subtracted individually for each data bin (cf. sect. 2.4).

${ }^{2}$ For reactions of type $a b \rightarrow 123$ the 23-helicity-frame ( $R 23$, $R$ indicates $R$ eference frame) is defined as the rest frame of the particles $(2,3)\left(\boldsymbol{p}_{3}=-\boldsymbol{p}_{2}\right)$. The respective (polar) helicity angle in this frame is defined as the angle between particle 3 and $1\left(\theta_{13}^{R 23}\right)$. By cyclic permutation three helicity frames can be constructed for the three-body final state $(R 23, R 31, R 12)$. 

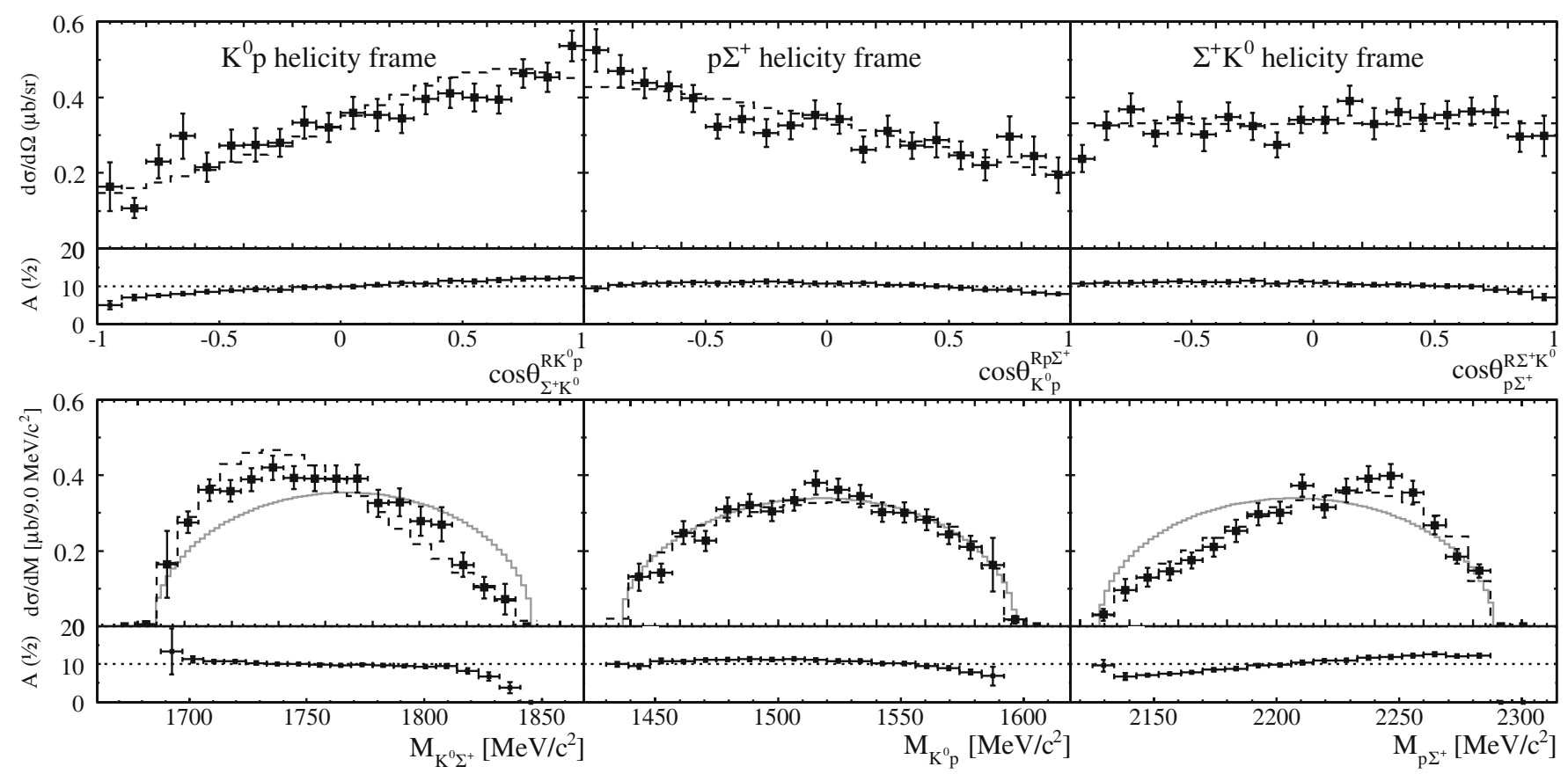

Fig. 7. Angular distributions of the particles in helicity frames (top) and the invariant-mass distributions for all three twobody subsystems (bottom) measured at an excess energy of $\epsilon=161$. Error bars for each data point are the square root of the quadratic sum of the statistical, acceptance, and signal-to-background-separation uncertainty. The dashed histograms represent the effect of an $N(1720,150)$ intermediate resonance, which is used as input for the Monte Carlo simulation. The grey curves show the distibutions expected for a pure phase-space scenario. Below each distribution the differential acceptance is shown. The numerical values of the differential cross-sections are listed in tables A.1 and A.2.

It is obvious that the distributions in the $p \Sigma^{+}$and $K^{0} p$ helicity frames are asymmetric $\left(\right.$ i.e. $\left.a_{1} \neq 0\right)$ while that in the $\Sigma^{+} K^{0}$ frame is almost isotropic $\left(a_{1} \approx 0\right)$. This can be infered quantitatively from the values of the Legendre polynomials coefficients listed in table 2 . It is also evident that the $K^{0} \Sigma^{+}$invariant-mass distribution deviates from that given by phase space (grey curve in fig. 7) and shows a clear enhancement towards smaller masses; thus, strongly advocating a production scenario involving a broad intermediate resonance with a central mass near (or below) the lower $K^{0} \Sigma^{+}$mass boundary.

The isotropic distribution observed in the $K^{0} \Sigma^{+}$helicity frame is of particular relevance in case of resonant production: Firstly it indicates that the decay channel of the resonance is $B^{*} \rightarrow K^{0} \Sigma^{+}$. Secondly, there is no preferred orientation of the $B^{*}$-spin with respect to the $B^{*}$ direction of flight. This is an indication of the presence of several partial waves. And thirdly, the observed isotropy signifies that only one resonance participates or, if more than one resonance is involved, they do not interfere (except for the possibility that the interference pattern mimics an isotropic distribution).

In the following, we assume that only one resonance participates. Indeed, all six distributions of fig. 7 are well described by the results of a Monte Carlo simulation (dashed histograms), where the three-particle final state is modeled via $p p \rightarrow p N^{*}, N^{*} \rightarrow K^{0} \Sigma^{+}$. The central mass and the Breit-Wigner width of the resonance were chosen to be $m_{N^{*}}=1720 \mathrm{MeV} / c^{2}$ and $\Gamma=150 \mathrm{MeV} / c^{2}$ (abbreviated in the following as $N(1720,150))$.
Table 2. Legendre polynomial coefficients (in units of $n \mathrm{~b} / \mathrm{sr}$ ) determined by least square fitting to angular distributions of the reaction $p p \rightarrow p K^{0} \Sigma^{+}$at $\epsilon=161 \mathrm{MeV}$, in the overall CMS, the Jackson and helicity frames (top to bottom).

\begin{tabular}{cccc}
\hline $\cos$ & $a_{0}$ & $a_{1}$ & $a_{2}$ \\
\hline$\theta_{p}^{*}$ & $335 \pm 20$ & $3 \pm 37$ & $59 \pm 39$ \\
$\theta_{K}^{*}$ & $330 \pm 22$ & $-10 \pm 44$ & $109 \pm 39$ \\
$\theta_{\Sigma^{+}}^{*}$ & $325 \pm 11$ & $22 \pm 20$ & $46 \pm 29$ \\
\hline$\theta_{b K}^{R K p}$ & $319 \pm 6$ & $38 \pm 12$ & $52 \pm 16$ \\
$\theta_{b p}^{R p \Sigma^{+}}$ & $322 \pm 7$ & $-27 \pm 14$ & $76 \pm 20$ \\
$\theta_{b \Sigma^{+}}^{R \Sigma^{+}}$ & $322 \pm 7$ & $-7 \pm 13$ & $99 \pm 16$ \\
\hline$\theta_{\Sigma^{+} K}^{R K p}$ & $323 \pm 9$ & $183 \pm 16$ & $-20 \pm 20$ \\
$\theta_{K p}^{R p \Sigma^{+}}$ & $326 \pm 9$ & $-130 \pm 17$ & $41 \pm 22$ \\
$\theta_{p \Sigma^{+}}^{R \Sigma^{+}}$ & $332 \pm 9$ & $19 \pm 17$ & $-19 \pm 22$ \\
\hline
\end{tabular}

The choice of this particular $N(1720,150)$ resulted from a series of Monte Carlo simulations which were performed to study the kinematical effect of mass and width of different resonances such as $N(1400,270)$ [15], $N(1650,300), \quad N(1720,150)$, and $N(1900,300)$ chosen from [49]. We also included an $N(1800,200)$ state in order to study the development of the kinematical effect with a narrower spacing of masses. (As kinematics does not de- 


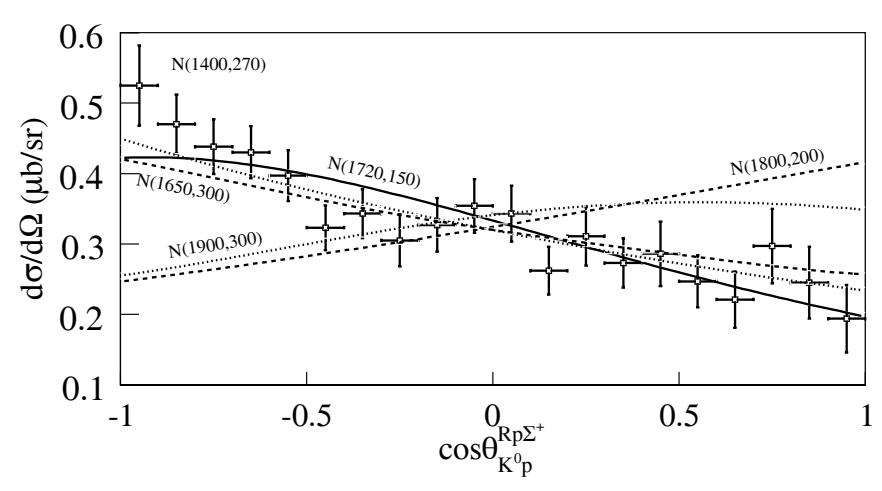

Fig. 8. Angular distribution measured in the $p \Sigma^{+}$helicity frame. The lines are the Monte Carlo result for various resonances $(N(m, \Gamma))$. Simulations with low mass resonances reproduce the measured data with similar quality, whereas heavy resonances clearly fail. The resonance with the best overall agreement between data and Monte Carlo is the $N(1720,150)$ which is plotted as solid line.

pend on isospin, the following statements apply also for $\Delta(1600), \Delta(1620), \Delta(1700), \Delta(1750)$, and $\Delta(1900)[49]$.

As a pictorial example of such an analysis the distributions obtained in the $p \Sigma^{+}$helicity helicity are shown in fig. 8. Due to kinematics, all heavy resonances have a positive slope in the $p \Sigma^{+}$helicity frame. They clearly fail to describe the data. In contrast, all distributions resulting from the decay of light resonances display negative slopes in this helicity frame and describe the data with similar accuracy.

In order to assess the quality of the overall description of the data by each of these $N^{*}$-resonances Monte Carlo simulations a simple quality criterion - root mean square of the error-weighted difference of data and Monte Carlo results, averaged over all bins for the six observables of fig. 7- was applied. It turns out that this figure of merit varies by about $10 \%$ for the three light resonances, where the $N(1720,150)$ yields the best averaged description. This resonance is used throughout the whole analysis as Monte Carlo input for acceptance correction.

The working hypothesis of a single resonance governing the $p K^{0} \Sigma^{+}$reaction process is further applied in the next subsection.

\subsection{Angular distributions in the CM and Jackson frames}

Angular distributions in the overall CMS can be described by Legendre polynomials. In the specific picture of a production mechanism involving an intermediate resonance $\left(p p \rightarrow p B^{*}\right)$ the CMS scattering angle of the proton directly reflects the angular momenta involved in the $p B^{*}$ channel. There is a linear dependence of the cosine of the scattering angle on momentum transfer $t_{1}$, thus the proton CMS angle can be taken as one of the linear independent variables in eq. (4). Due to the symmetric $p p$ entrance channel all CMS distributions must of course be symmetric with respect to $\cos \theta^{*}=0$.

Jackson frames ${ }^{3}$ are the natural Lorentz invariant frames to investigate the angular momenta involved in a two-particle subsystem [56]. To illustrate this, consider the secondary decay $\left(B^{*} \rightarrow K^{0} \Sigma^{+}\right)$at the $\pi p \rightarrow K^{0} \Sigma^{+}$ vertex in fig. $1 \mathrm{~b}$, c; this represents a " $2 \rightarrow 2$ " reaction. In this picture, the inverse reaction $\left(K^{0} \Sigma^{+} \rightarrow p \pi\right)$ must have the same properties due to time reversal invariance. If one now imagines colliding beams of kaons and hyperons with $\boldsymbol{p}_{Y}=-\boldsymbol{p}_{K}$, which is by definition the $K Y$ Jackson frame, it is self-evident that the distribution of angles $\theta_{b K}^{R K Y}$ of the (in this case emerging) proton with respect to the (in this case beam-axis defining) kaon contains information on the relative angular momenta involved. There is a linear relation between $t_{2}$ and $\cos \theta_{b \Sigma^{+}}^{R K^{0} \Sigma^{+}}$, i.e. the $K^{0} \Sigma^{+}$Jackson angle can substitute the momentum transfer between the beam particle and one of the resonance decay particles in eq. (4). It is important to notice that the Jackson frame is a different Lorentz frame than the CMS and no symmetry with respect to $\cos \theta=0$ is required. However, the distributions measured with respect to the target and beam proton must be identical.

The angular distributions of all primary particles in the CMS measured at an excess energy of $\epsilon=161 \mathrm{MeV}$ are shown in the upper row of fig. 9. The lower row shows the angular distributions in all three Jackson frames. The variation of the acceptance is shown under each distribution. In case of the proton and kaon the acceptance shows a quite strong angular dependence which is due to the strongly decreasing probability of the kaon to reach the decay volume when emitted further into the backward CMS hemisphere $\left(\cos \theta_{K^{0}}^{*} \rightarrow-1\right)$. This experimental effect is mirrored in the proton acceptance distribution, as protons and kaon tend to be emitted into opposite hemispheres. For the hyperon, constructed from the combined momentum vectors of kaon and proton, a much weaker angular dependence of its acceptance is found. Nevertheless, after acceptance correction all spectra show the necessary symmetry with respect to $\cos \theta^{*}=0\left(a_{1}=0\right)$, as the values of $a_{1}$ given in table 2 are all compatible with zero within uncertainty. The $a_{2} / a_{0}$ ratio changes by less than $7 \%$ if $a_{1}$ is forced to be zero.

A clear anisotropy is observed for all Jackson-frame distributions, as indicated by the $a_{1}$ coefficients listed in table 2. There is no need to introduce Legendre polynomials of order higher than two. In the picture of a two step production process, this finding for the proton CMS angular distribution and the distribution in the $K^{0} \Sigma^{+}$

3 For reactions of type $a b \rightarrow 123$ the Jackson frame is defined as the Lorentz frame in which the center of mass of the particles $(2,3)$ is at rest $\left(\boldsymbol{p}_{3}=-\boldsymbol{p}_{2}\right)$. The 23-Jackson frame and the 23-helicity frame are in fact the same Lorentz frame (R23). The word "frame" rather refers to the choice of the reference axis which in case of Jackson frames is the direction of the beam particle. The Jackson angle is then defined as the angle between the beam direction and that of particle $3\left(\theta_{b 3}^{R 23}\right)$. By cyclic permutation three Jackson frames can be constructed for the three-body final state $(R 23, R 31, R 12)$. 


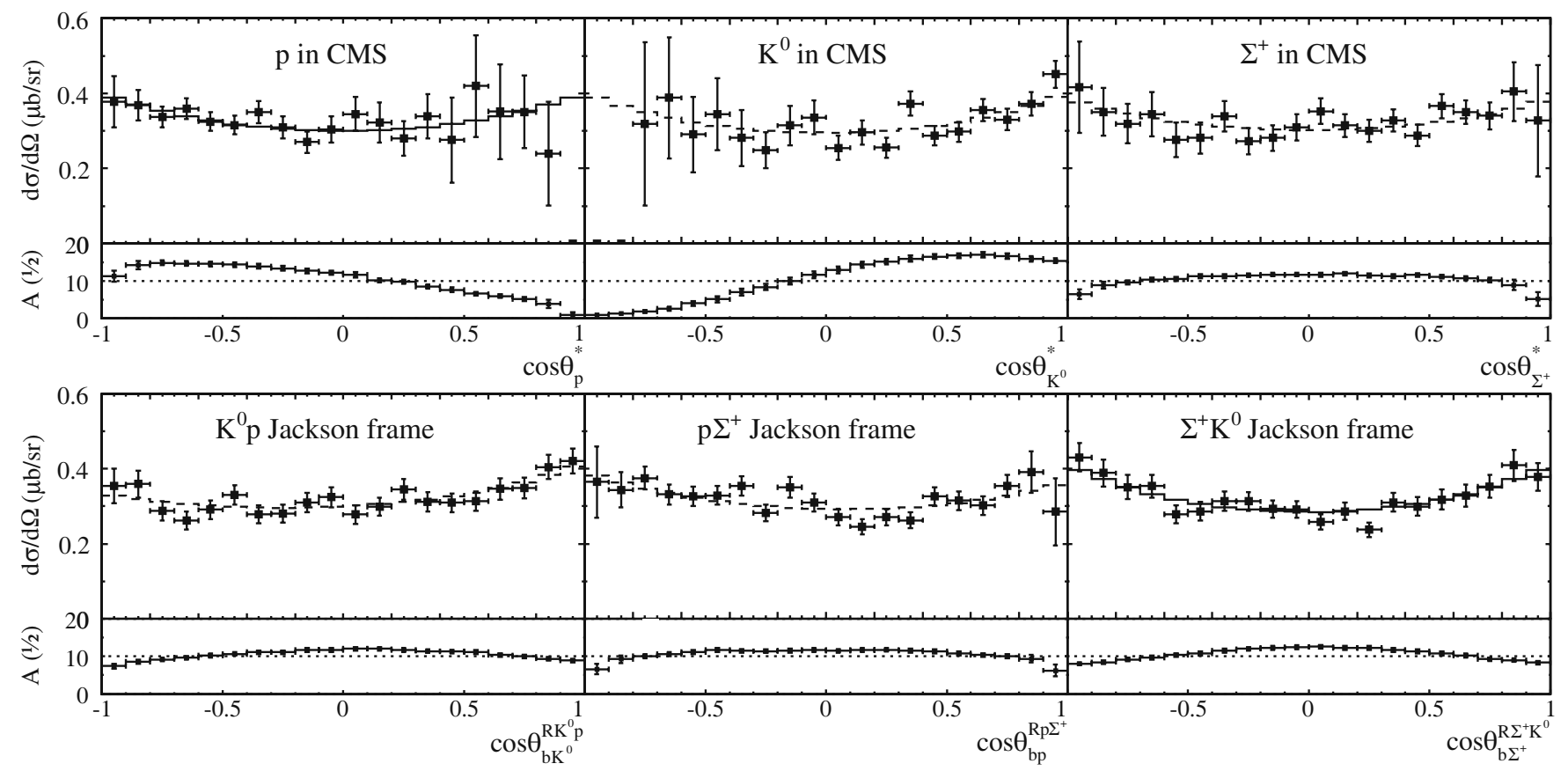

Fig. 9. Angular distributions of the particles in the overall CMS (top) and the Jackson frames (bottom), for the reaction $p p \rightarrow p K^{0} \Sigma^{+}$measured at an excess energy of $\epsilon=161 \mathrm{MeV}\left(p_{\text {beam }}=3059 \mathrm{MeV} / c\right)$. Error bars for each data point are the square root of the quadratic sum of the statistical, acceptance, and signal-to-background-separation uncertainty. The solid histogram in the proton CMS and $\Sigma^{+} K^{0}$ Jackson frame represents the respective Legendre polynomials of table 2 which are used as MC filter. Their affect on all other angular distributions are shown by the dashed histograms. Below each angular distributions the differential acceptance is shown. The numerical values of the differential cross-sections are listed in table A.2.

Jackson frame indicates that angular momenta of $l \leq 1$ are involved at both the $p p \rightarrow p B^{*}$ and $B^{*} \rightarrow K^{0} \Sigma^{+}$ subprocess.

In order to corroborate the two step production scenario we now turn to the simultaneous description of the data by Monte Carlo simulations, the result of which is shown as either solid or dashed histograms in fig. 9. The solid histograms shown represent Monte Carlo data which were filtered by means of weight functions in the two natural frames described above: The first filter was tailored to reproduce the measured proton CMS distribution (solid line in fig. 9, upper left frame). This filter significantly affects via kinematic correlation the two Jackson frames containing the proton (distribution in the $p K^{0}$ and $p \Sigma^{+}$ Jackson frames, dashed line in fig. 9, lower left and lower middle frame), while barely affecting the kaon and hyperon CMS distributions. The angular distribution in the $K^{0} \Sigma^{+}$Jackson frame is not affected at all. Hence it is justified to apply a second filter which is tailored to reproduce the measured distribution in the $\Sigma^{+} K^{0}$ Jackson frame (solid line in fig. 9, lower right frame). This filter significantly affects via kinematic correlations the kaon and hyperon CMS distributions (dashed lines in fig. 9, uppermiddle and upper-right frame) while barely affecting the distribution in the $p \Sigma^{+}$and $p K^{0}$ Jackson frames. The proton angular distribution in the CMS is not affected at all. The observation that both filters do not effect each other is a consequence of the linear independence of $t_{1}$ and $t_{2}$.

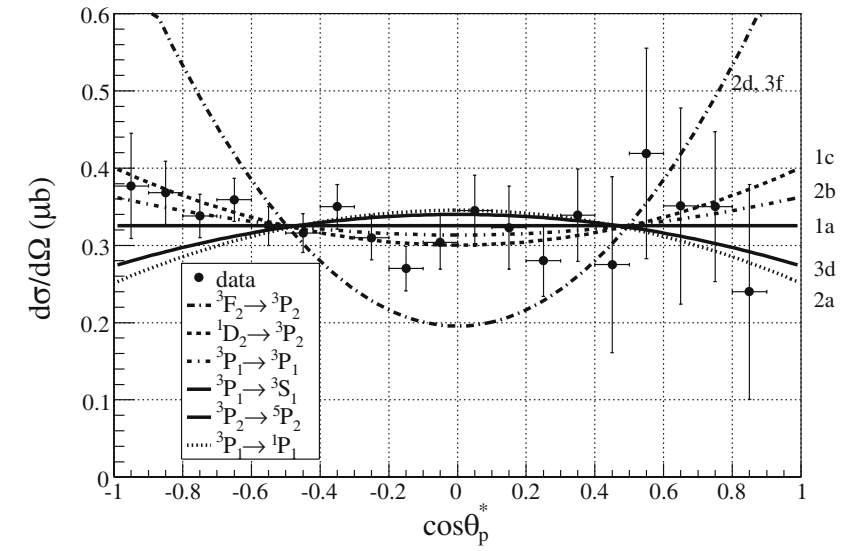

Fig. 10. Selected theoretical angular distributions calculated for the $p p \rightarrow p N^{*}$ reaction $\left(N^{*}: S_{11}, P_{11}, P_{13}\right)$ according to [57]. All distributions are of the type $\mathrm{d} \sigma / \mathrm{d} \Omega=a_{0}+$ $a_{2} P_{2}(\cos \theta)$, i.e. $l_{p N^{*}} \leq 1$.

Two other filter combinations also fulfill the requirement of linear independence $\left(K^{0}\right.$-CMS and $p \Sigma^{+}$-Jackson frame; $\Sigma^{+}$-CMS and $p K^{0}$-Jackson frame). Although these filter combinations represent somewhat awkward reaction scenarios (i.e. $p p \rightarrow K^{0} X, X \rightarrow p \Sigma^{+}$and $p p \rightarrow \Sigma^{+} X$, $\left.X \rightarrow p K^{0}\right)$ they are not excluded a priori. Therefore both 
Table 3. Possible transitions for $p p \rightarrow p N\left(S_{11}\right), p N\left(P_{11}\right), p N\left(P_{13}\right)$ which yield differential cross-sections described by $\mathrm{d} \sigma / \mathrm{d} \Omega=$ $a_{0}+a_{2} P_{2}(\cos \theta)$ with all $a_{2}$ coefficients being $\neq 0$ but those of transition 1a and 3e which have $a_{2}=0$. Note that initial partial waves of type ${ }^{1} S_{0}$ and ${ }^{3} P_{0}$ result in isotropic angular distributions irrespectively of the intermediate resonance.

\begin{tabular}{|c|c|c|c|c|c|c|c|c|}
\hline & & $\mathrm{a}$ & $\mathrm{b}$ & $\mathrm{c}$ & $\mathrm{d}$ & e & $\mathrm{f}$ & $\mathrm{g}$ \\
\hline 1 & $p p \rightarrow p S_{11}$ & ${ }^{3} P_{1} \rightarrow{ }^{3} S_{1}$ & ${ }^{3} P_{1} \rightarrow{ }^{3} D_{1}$ & ${ }^{1} D_{2} \rightarrow{ }^{3} P_{2}$ & & & & \\
\hline 2 & $p p \rightarrow p P_{11}$ & ${ }^{3} P_{1} \rightarrow{ }^{1} P_{1}$ & ${ }^{3} P_{1} \rightarrow{ }^{3} P_{1}$ & ${ }^{3} P_{2} \rightarrow{ }^{3} P_{2}$ & ${ }^{3} F_{2} \rightarrow{ }^{3} P_{2}$ & & & \\
\hline 3 & $p p \rightarrow p P_{13}$ & ${ }^{3} P_{1} \rightarrow{ }^{3} P_{1}$ & ${ }^{1} P_{1} \rightarrow{ }^{5} P_{1}$ & ${ }^{3} P_{2} \rightarrow{ }^{3} P_{2}$ & ${ }^{3} P_{2} \rightarrow{ }^{5} P_{2}$ & ${ }^{1} D_{2} \rightarrow{ }^{5} S_{2}$ & ${ }^{3} F_{2} \rightarrow{ }^{3} P_{2}$ & ${ }^{3} F_{2} \rightarrow{ }^{5} P_{2}$ \\
\hline
\end{tabular}

combinations were tested for the sake of completeness with the method introduced in sect. 3.3. Both filter combinations yield a description of the data slightly inferior to the filter combination discussed above (the ones shown as solid histograms in fig. 9).

In the following it is assumed that both the distribution in the $K^{0} \Sigma^{+}$Jackson frame and that of the proton in the overall CMS reflect the physical cause and the other four distributions are simply their kinematical reflections. In addition the discussion will be based on the properties of resonant $K^{0} \Sigma^{+}$production from an initial state of identical particles. The angular distribution in the Jackson frame reveals that the $K^{0} \Sigma^{+}$subsystem carries angular momentum of $l \leq 1$ (cf. table 2 ).

It then follows from isospin, angular momentum, and parity conservation that only nucleon resonances of the type $S_{11}, P_{11}$, and $P_{13}$ and $\Delta$-resonances of the type $S_{31}$, $P_{31}$, and $P_{33}$ can be involved. All other resonances have decay angular momenta of $l>1$.

In the next step, all angular distributions for protons in the CMS are calculated for $p p \rightarrow p N\left(S_{11}\right), p N\left(P_{11}\right)$, and $p N\left(P_{13}\right)$ applying the formalism of Blatt and Biedenharn [57]. Entrance channel partial waves up to ${ }^{3} F_{4}$ are considered (spectroscopic notation ${ }^{2 S+1} L_{J}$, with $S$ : channel spin, $L$ : orbital angular momentum of the two-body system, $J$ : total angular momentum of the channel). Angular distributions arising from ${ }^{1} S_{0}$ and ${ }^{3} P_{0}$ entrance channel partial waves are isotropic irrespective of the final state due to $J=0$ for the entrance channel.

All other transitions are listed in table 3. Interestingly, all angular distributions resulting from these transitions are of the type $\mathrm{d} \sigma / \mathrm{d} \Omega=a_{0}+a_{2} P_{2}\left(\cos \theta_{C M}\right)$, i.e. no term higher than $P_{2}$ is involved which is perfectly in line with the experimental finding of $l \leq 1$. The calculations according to ref. [57] yield $a_{2}=\overline{0}$ for transition $1 \mathrm{a}$ and $3 \mathrm{e}$ (isotropy), $a_{2}>0$ (concave shape) for $1 \mathrm{c}, 2 \mathrm{~b}, 2 \mathrm{c}, 2 \mathrm{~d}, 3 \mathrm{a}$, $3 \mathrm{c}$, 3f and $a_{2}<0$ (convex shape) for $1 \mathrm{~b}, 2 \mathrm{a}, 3 \mathrm{~b}, 3 \mathrm{~d}, 3 \mathrm{~g}$.

In the case of $\Delta$-resonances isospin selectivity requires their production from a spin singlet state (isospin triplet state) in the entrance channel, thus ruling out entrance channel $P$ - or $F$-wave contributions. It is found that only two transitions are possible which yield angular distributions with $l \leq 1$ namely $\left({ }^{1} D_{2}\right)_{i} \rightarrow\left({ }^{3} P_{2}\right)_{f}$ involving the $\Delta\left(S_{31}\right)$ intermediate state and $\left({ }^{1} D_{2}\right)_{i} \rightarrow\left({ }^{5} S_{2}\right)_{f}$ via the $\Delta\left(P_{33}\right)$, the latter being isotropic. A $\Delta\left(P_{31}\right)$-resonance yields angular distributions with an $a_{4} P_{4}$ term indicative of $l=2$ which is not observed; hence such a resonance is excluded from the further discussion.

Examples of calculated distributions resulting from transitions listed in table 3 are shown in fig. 10 normalized to the experimental data. The concave curves $1 \mathrm{c}$ and $2 \mathrm{~b}$ match the data best. However, it is very unlikely that only one or two specific transitions govern the reaction process. In fact, an inspection of table 3 reveals that the same initial and final state partial waves can occur, however with different intermediate resonances being involved (e.g., 2c$3 \mathrm{c}, 2 \mathrm{~d}-3 \mathrm{f})$. In addition, various initial state partial waves pass through a particular resonance but end up in different final-state partial waves, (e.g., $2 \mathrm{a}-2 \mathrm{~b}, 3 \mathrm{c}-3 \mathrm{~d})$. A rather complicated scenario turns up and it is therefore impossible to pin down the individual contributions of particular transitions within this simple reaction model. The question of which partial waves dominate the reaction can only be answered by a complete partial wave analysis.

Nevertheless, fig. 10 indicates that a proper superposition of transitions will match the experiment data. Thus, the working hypothesis introduced in sect. 3.3 of a reaction mechanism involving an intermediate resonance is now limited to the states $S_{11}, P_{11}, P_{13}, S_{31}$, and $P_{33}$.

The clear non-zero angular momentum observed in the $K^{0} \Sigma^{+}$Jackson frame requires considerable strength of participating resonances with intrinsic angular momentum, i.e. favoring $P_{11}$ and/or $P_{13}$ and/or $P_{33}$. Resonances with these spins and parities can be related to those listed by the PDG. It turns out that $N(1710) P_{11}$, $N(1720) P_{13}$, and $\Delta(1600) P_{33}$ are the only candidates with appropriate mass and a $K \Sigma$ decay branch. Exactly this type of resonance $(m \approx 1720 \mathrm{MeV}, \Gamma \approx 150 \mathrm{MeV}$ or $m \approx 1650 \mathrm{MeV}, \Gamma \approx 300 \mathrm{MeV}$ ) was also favored in describing the invariant-mass and helicity angular distributions. As no $\Delta\left(S_{31}\right)$-resonance is known in this mass range which decays into $K \Sigma$, the $N(1650) S_{11}$ is the only further intermediate resonance to be possibly involved.

It must be emphazised that the results for mass and width of a potential intermediate resonance obtained in sect. 3.3 and in this section resulted from exploiting orthogonal parameter spaces, namely $s_{1}, s_{2}, s_{3}$ and $t_{1}, t_{2}$, respectively. The possibility of cross-checking results obtained in various reference frames and their combined interpretation underlines the importance of an analysis of the full four-dimensional reaction space. 


\section{Summary}

The reaction $p p \rightarrow p K^{0} \Sigma^{+}$was studied at the excess energies of $\epsilon=126,161$ and $206 \mathrm{MeV}$ with the COSYTOF spectrometer. The large acceptance allows the fourfold differential cross-section $\mathrm{d}^{4} \sigma / \mathrm{d} s_{1} \mathrm{~d} s_{2} \mathrm{~d} t_{1} \mathrm{~d} t_{2}$ to be determined. This high dimensionality however, needs to be reduced by projecting onto a reduced number of observables to highlight certain aspects of this four-dimensional parameter space. In doing so, Dalitz plots, invariant mass spectra, angular distributions in helicity frames, the overall CMS, and Jackson frames are obtained for $\epsilon=161 \mathrm{MeV}$

The measured total cross-sections are the first to build the excitation function between the production threshold and the energy region covered by early bubble chamber experiments. The observed increase of the total cross-section with excess energy is consistent with the increase of phasespace volume. This, however, does not mean that the reaction is governed by phase space; it rather implies that the transition matrix element does not (strongly) depend on energy up to about $\approx 200 \mathrm{MeV}$. The bubble chamber data show the total cross-section to be rather constant $(\approx 20 \mu \mathrm{b})$ in the energy range from 300 to $1500 \mathrm{MeV}$.

The Dalitz plots do not show any sign of final-state interactions. However, a clear signal for resonant contributions $\left(p p \rightarrow p B^{*} ; B^{*}=N^{*}, \Delta^{*} ; B^{*} \rightarrow K^{0} \Sigma^{+}\right)$is found near the lower $K^{0} \Sigma^{+}$mass boundary. This qualitative result is confirmed by the quantitative analysis of invariant-mass spectra and angular distributions in helicity frames. The best description of the data by Monte Carlo is found for resonances with $m_{N^{*}} \approx 1720 \mathrm{MeV} / c^{2}$ and $\Gamma \approx 150 \mathrm{MeV} / c^{2}$. However, a contribution of lighter $N^{*}$ or $\Delta^{*}$-resonances cannot be excluded. In contrast, the data clearly rule out a significant contribution of resonances with masses larger than $1800 \mathrm{MeV} / c^{2}$. The isotropic distribution in the $K^{0} \Sigma^{+}$helicity frame shows again the absence of $p \Sigma^{+}$final-state interactions as well as the absence of interference effects which would distort this distribution. The absence of interference allows the production mechanism to be interpreted as a two-step process $p p \rightarrow p B^{*}, B^{*} \rightarrow K^{0} \Sigma^{+}$involving only one resonance.

The decay angular momentum of the resonance is reflected in the $K^{0} \Sigma^{+}$Jackson frame angular distribution and was found to be $l \leq 1$. Parity and angular momentum conservation then restrict the possible states to be $S_{11}$, $P_{11}, P_{13}\left(N^{*}\right)$ or $P_{33}\left(\Delta^{*}\right)$. The shape of proton angular distributions in the $\mathrm{CM}$ system for the $p p \rightarrow p B^{*}$ reaction was calculated under the assumption that one of these resonances is involved. It turned out that all these calculated distributions have a relative angular momentum in the $p$ $B^{*}$ system of $l \leq 1$ in agreement with that deduced from the experimental proton CMS distribution. Considering that the angular distribution in the $K^{0} \Sigma^{+}$Jackson frame is clearly anisotropic and in view of the conclusions drawn from inspecting the distributions of invariant masses and helicity angles, $N(1710) P_{11}$ and/or $N(1720) P_{13}$ are the remaining candidate(s) for the dominating process of the $p p \rightarrow p K^{0} \Sigma^{+}$reaction. Contributions from $N(1650) S_{11}$ and/or $\Delta(1600) P_{33}$ could also be present.
A simultaneous description of all 12 differential distributions measured was possible by assuming a single resonance $\left(N(1720), \Gamma=150 \mathrm{MeV} / c^{2}\right)$ to dominate the reaction and applying weight functions (filters) on both the proton CMS angular distribution and the distribution in the $K^{0} \Sigma^{+}$Jackson frame. These kinematic constraints are linearly independent in the four-dimensional reaction space. Hence, this simple approach to describe the reaction mechanism is self-consistent.

An advanced analysis of the data could be based on a partial-wave analysis as performed by the Bonn-Gatchina group for various other reactions [58]. This might stimulate the further development of theoretical models which describe the associated strangeness production in protonproton collisions from first principles.

The authors would like to express their gratitude to the COSY staff for the operation of the accelerator during the experiments. This work was supported in part by grants from BMBF and Forschungszentrum Jülich (COSY-FFE).

\section{Appendix A. Datatables}

Table A.1. Differential cross-sections (in units of $\left.\mathrm{nb} /\left(9.0 \mathrm{MeV} / c^{2}\right)\right)$ of the invariant-mass distributions shown in fig. 7 .

\begin{tabular}{cccccc}
\hline$m_{K \Sigma^{+}}$ & $\mathrm{d} \sigma / \mathrm{d} m$ & $m_{K p}$ & $\mathrm{~d} \sigma / \mathrm{d} m$ & $m_{p \Sigma^{+}}$ & $\mathrm{d} \sigma / \mathrm{d} m$ \\
\hline 1674.50 & - & 1434.50 & - & 2129.50 & $31 \pm 15.1$ \\
1683.50 & $6 \pm 3.6$ & 1443.50 & $131 \pm 36.1$ & 2138.50 & $97 \pm 28.0$ \\
1692.50 & $164 \pm 88.7$ & 1452.50 & $141 \pm 24.6$ & 2147.50 & $130 \pm 24.9$ \\
1701.50 & $276 \pm 29.2$ & 1461.50 & $248 \pm 29.8$ & 2156.50 & $146 \pm 24.8$ \\
1710.50 & $361 \pm 28.1$ & 1470.50 & $227 \pm 26.5$ & 2165.50 & $175 \pm 21.5$ \\
1719.50 & $358 \pm 28.3$ & 1479.50 & $310 \pm 31.3$ & 2174.50 & $210 \pm 25.1$ \\
1728.50 & $389 \pm 30.0$ & 1488.50 & $321 \pm 29.7$ & 2183.50 & $253 \pm 29.6$ \\
1737.50 & $420 \pm 32.8$ & 1497.50 & $305 \pm 27.2$ & 2192.50 & $297 \pm 29.1$ \\
1746.50 & $394 \pm 31.0$ & 1506.50 & $334 \pm 28.2$ & 2201.50 & $301 \pm 29.1$ \\
1755.50 & $391 \pm 34.2$ & 1515.50 & $380 \pm 31.3$ & 2210.50 & $373 \pm 29.6$ \\
1764.50 & $391 \pm 35.5$ & 1524.50 & $362 \pm 28.7$ & 2219.50 & $315 \pm 27.9$ \\
1773.50 & $391 \pm 36.9$ & 1533.50 & $345 \pm 29.8$ & 2228.50 & $360 \pm 30.9$ \\
1782.50 & $326 \pm 35.7$ & 1542.50 & $303 \pm 26.2$ & 2237.50 & $392 \pm 32.2$ \\
1791.50 & $328 \pm 36.9$ & 1551.50 & $301 \pm 26.5$ & 2246.50 & $398 \pm 31.0$ \\
1800.50 & $279 \pm 38.9$ & 1560.50 & $281 \pm 27.6$ & 2255.50 & $353 \pm 31.4$ \\
1809.50 & $270 \pm 45.2$ & 1569.50 & $244 \pm 26.7$ & 2264.50 & $268 \pm 24.1$ \\
1818.50 & $163 \pm 32.5$ & 1578.50 & $210 \pm 29.6$ & 2273.50 & $185 \pm 19.6$ \\
1827.50 & $103 \pm 28.3$ & 1587.50 & $163 \pm 71.3$ & 2282.50 & $147 \pm 17.6$ \\
1836.50 & $73 \pm 40.9$ & 1596.50 & $18 \pm 9.5$ & 2291.50 & - \\
1845.50 & - & 1605.50 & - & 2300.50 & - \\
\hline & & & & &
\end{tabular}


Table A.2. Differential cross-sections (in units of nb/sr) of the angular distributions shown in figs. 7 and 9 .

\begin{tabular}{|c|c|c|c|c|c|c|c|c|c|}
\hline $\cos$ & $\theta_{p}^{*}$ & $\theta_{K}^{*}$ & $\theta_{\Sigma^{+}}^{*}$ & $\theta_{b p}^{R p \Sigma^{+}}$ & $\theta_{b K}^{R K p}$ & $\theta_{b \Sigma^{+}}^{R \Sigma^{+} K}$ & $\theta_{K p}^{R p \Sigma^{+}}$ & $\theta_{\Sigma^{+} K}^{R K p}$ & $\theta_{p \Sigma^{+}}^{R \Sigma^{+} K}$ \\
\hline-0.95 & $377 \pm 68$ & - & $416 \pm 122$ & $354 \pm 46$ & $365 \pm 94$ & $430 \pm 38$ & $163 \pm 65$ & $525 \pm 57$ & $238 \pm 60$ \\
\hline-0.85 & $368 \pm 41$ & - & $351 \pm 64$ & $359 \pm 36$ & $344 \pm 48$ & $388 \pm 36$ & $107 \pm 27$ & $470 \pm 42$ & $325 \pm 38$ \\
\hline-0.75 & $338 \pm 28$ & $319 \pm 218$ & $319 \pm 53$ & $288 \pm 26$ & $375 \pm 31$ & $351 \pm 32$ & $231 \pm 44$ & $438 \pm 39$ & $368 \pm 44$ \\
\hline-0.65 & $359 \pm 28$ & $388 \pm 161$ & $345 \pm 59$ & $262 \pm 24$ & $331 \pm 26$ & $353 \pm 31$ & $298 \pm 60$ & $430 \pm 37$ & $304 \pm 34$ \\
\hline-0.55 & $325 \pm 25$ & $290 \pm 101$ & $276 \pm 46$ & $291 \pm 25$ & $327 \pm 25$ & $278 \pm 24$ & $215 \pm 39$ & $397 \pm 36$ & $346 \pm 43$ \\
\hline-0.45 & $316 \pm 25$ & $345 \pm 96$ & $281 \pm 41$ & $330 \pm 26$ & $329 \pm 25$ & $287 \pm 25$ & $273 \pm 42$ & $323 \pm 32$ & $301 \pm 40$ \\
\hline-0.35 & $350 \pm 29$ & $281 \pm 74$ & $339 \pm 40$ & $278 \pm 24$ & $354 \pm 26$ & $314 \pm 26$ & $274 \pm 44$ & $343 \pm 35$ & $349 \pm 37$ \\
\hline-0.25 & $310 \pm 29$ & $248 \pm 48$ & $273 \pm 35$ & $280 \pm 24$ & $282 \pm 22$ & $313 \pm 25$ & $280 \pm 38$ & $305 \pm 37$ & $324 \pm 36$ \\
\hline-0.15 & $270 \pm 29$ & $314 \pm 52$ & $281 \pm 34$ & $310 \pm 25$ & $351 \pm 27$ & $293 \pm 23$ & $334 \pm 42$ & $327 \pm 38$ & $274 \pm 33$ \\
\hline-0.05 & $304 \pm 35$ & $336 \pm 45$ & $310 \pm 35$ & $325 \pm 27$ & $310 \pm 24$ & $292 \pm 22$ & $320 \pm 39$ & $354 \pm 38$ & $341 \pm 35$ \\
\hline 0.05 & $345 \pm 46$ & $255 \pm 33$ & $352 \pm 34$ & $278 \pm 25$ & $271 \pm 23$ & $258 \pm 21$ & $359 \pm 42$ & $343 \pm 40$ & $340 \pm 35$ \\
\hline 0.15 & $323 \pm 54$ & $296 \pm 32$ & $314 \pm 30$ & $299 \pm 24$ & $246 \pm 20$ & $286 \pm 23$ & $354 \pm 43$ & $262 \pm 34$ & $391 \pm 40$ \\
\hline 0.25 & $280 \pm 46$ & $255 \pm 27$ & $300 \pm 29$ & $345 \pm 28$ & $270 \pm 21$ & $237 \pm 20$ & $344 \pm 38$ & $311 \pm 42$ & $331 \pm 41$ \\
\hline 0.35 & $339 \pm 60$ & $373 \pm 33$ & $328 \pm 30$ & $312 \pm 25$ & $263 \pm 22$ & $311 \pm 25$ & $396 \pm 40$ & $273 \pm 35$ & $361 \pm 36$ \\
\hline 0.45 & $275 \pm 114$ & $288 \pm 26$ & $288 \pm 29$ & $310 \pm 25$ & $326 \pm 24$ & $300 \pm 24$ & $412 \pm 39$ & $286 \pm 46$ & $347 \pm 36$ \\
\hline 0.55 & $419 \pm 136$ & $298 \pm 27$ & $366 \pm 32$ & $313 \pm 26$ & $315 \pm 25$ & $318 \pm 27$ & $400 \pm 37$ & $247 \pm 37$ & $354 \pm 37$ \\
\hline 0.65 & $351 \pm 127$ & $356 \pm 29$ & $350 \pm 32$ & $346 \pm 29$ & $302 \pm 24$ & $329 \pm 29$ & $394 \pm 38$ & $221 \pm 40$ & $363 \pm 37$ \\
\hline 0.75 & $350 \pm 97$ & $331 \pm 28$ & $340 \pm 36$ & $348 \pm 28$ & $354 \pm 30$ & $353 \pm 31$ & $464 \pm 38$ & $297 \pm 53$ & $361 \pm 42$ \\
\hline 0.85 & $240 \pm 139$ & $373 \pm 31$ & $405 \pm 78$ & $404 \pm 33$ & $391 \pm 56$ & $409 \pm 42$ & $453 \pm 39$ & $245 \pm 51$ & $296 \pm 40$ \\
\hline 0.95 & - & $451 \pm 36$ & $328 \pm 148$ & $421 \pm 34$ & $285 \pm 89$ & $378 \pm 37$ & $537 \pm 40$ & $194 \pm 48$ & $298 \pm 66$ \\
\hline
\end{tabular}

Open Access This is an open access article distributed under the terms of the Creative Commons Attribution License (http://creativecommons.org/licenses/by/2.0), which permits unrestricted use, distribution, and reproduction in any medium, provided the original work is properly cited.

\section{References}

1. D. Grzonka, K. Kilian, Nucl. Phys. A 626, 41 (1997).

2. J.T. Balewski et al., Nucl. Phys. A 626, 85 (1997).

3. J.T. Balewski et al., Phys. Lett. B 420, 211 (1998).

4. S. Severin et al., Phys. Rev. Lett. 83, 682 (1999).

5. P. Kowina et al., Eur. Phys. J. A 22, 293 (2004).

6. M. Abd El-Samad et al., Phys. Lett. B 632, 27 (2006).

7. T. Rożek et al., Phys. Lett. B 643, 251 (2006).

8. Yu. Valdau et al., Phys. Lett.B 652, 245 (2007).

9. M. Abd El-Samad et al., Phys. Lett. B 688, 142 (2010).

10. S. Abdel-Samad et al., Eur. Phys. J. A 46, 27 (2010).

11. Yu. Valdau et al., Phys. Rev. C 81, 045208 (2010).

12. A. Budzanowski et al., Phys. Lett. B 692, 10 (2010).

13. A. Sibirtsev et al., Eur. Phys. J. A 27, 269 (2006).

14. A. Sibirtsev et al., Eur. Phys. J. A 29, 363 (2006).

15. B.C. Lui, B.S. Zou, Phys. Rev. Lett. 96, 042002 (2006).

16. A. Gasparin et al., Phys. Lett. B 480, 273 (2000).

17. M. Dillig, M. Schott, arXiv:nucl-th/0604059v1.

18. J.M. Laget, Phys. Lett. B 259, 24 (1991).

19. J.M. Laget, Nucl. Phys. A 691, 11 (2001).

20. Yu. Valdau, C. Wilkin, Phys. Lett. B 696, 23 (2011).

21. M. Abdel-Bary et al., Phys. Lett. B 649, 252 (2007).
22. R.I. Louttit et al., Phys. Rev. 123, 1465 (1961).

23. E. Bierman et al., Phys. Rev. 147, 922 (1966).

24. G. Alexander et al., Phys. Rev. 154, 1284 (1967).

25. S.O. Holmgren et al., Nuovo Cimento A 51, 305 (1968).

26. I. Sondhi et al., Phys. Lett. B 26, 645 (1968).

27. W. Chinowsky et al., Phys. Rev. 165, 1466 (1968).

28. M.W. Firebaugh et al., Phys. Rev. 172, 1354 (1968).

29. W.M. Dunwoodie, PhD thesis, UCLA 1033 (1968).

30. A. Baldini et al., Landolt-Börnstein: Numerical Data and Functional Relationships in Science and Technology, New Series, Vol. I/12b (Springer, New York, Heidelberg, 1988).

31. E. Ferrari, Phys. Rev. 120, 988 (1960).

32. Tsu Yao, Phys. Rev. 125, 1048 (1962).

33. E. Ferrari, S. Serio, Phys. Rev. 167, 1298 (1968).

34. C.B. Dover, Brookhaven National Laboratory BNL84595, DE93 010879 (1993).

35. A. Sibirtsev et al., Nucl. Phys. A 646, 427 (1999).

36. K. Tsushima et al., Phys. Rev. C 59, 369 (1999).

37. M. Abdel-Bary et al., Phys. Lett. B 647, 351 (2007).

38. M. Abdel-Bary et al., Eur. Phys. J. A 44, 7 (2010).

39. M. Schulte-Wissermann, PhD Thesis, TU Dresden (2004).

40. M. Dahmen, PhD Thesis, Rheinische Friedrich-WilhelmUniversität Bonn, Jül-3140, ISSN 0944-2952 (1995).

41. R. Bilger et al., Phys. Lett. B 420, 217 (1998).

42. A. Hassan et al., Nucl. Instrum. Methods A 425, 403 (1999).

43. M. Dahmen et al., Nucl. Instrum. Methods A 348, 97 (1994).

44. A. Böhm et al., Nucl. Instrum. Methods A 443, 238 (2000).

45. M. Abdel-Bary et al., Phys. Lett. B 662, 14 (2008).

46. R. Bilger et al., Phys. Lett. B 429, 195 (1998). 
47. M. Abdel-Bary et al., Eur. Phys. J. A 16, 127 (2003).

48. L. Karsch, PhD Thesis, TU Dresden (2005).

49. Particle Data Group (W.-M. Yao et al.), J. Phys. G 33, 1 (2006).

50. S. Brand, PhD Thesis, Ruhr-Universität Bochum (1995).

51. U. Zielinsky, PhD Thesis, Ruhr-Universität Bochum (1999).

52. D. Albers et al., Phys. Rev. Lett. 78, 1652 (1997).
53. E. Byckling, K. Kajantie, Particle Kinematics (John Wiley \& Sons, 1973).

54. E.L. Berger, Phys. Rev. Lett. 23, 1139 (1969).

55. R.H. Dalitz, Philos. Mag. Series 7 44, 1068 (1953).

56. K. Gottfried, J.D. Jackson, Nuovo Cimento 33, 309 (1964).

57. J.M. Blatt, L.C. Biedenharn, Rev. Mod. Phys. 24, 258 (1952).

58. A.V.Sarantsev et al., Eur. Phys. J. A 25, 441 (2005). 